\title{
ISOPARAMETRIC FOLIATIONS, A PROBLEM OF EELLS-LEMAIRE AND CONJECTURES OF LEUNG
}

\author{
CHAO QIAN AND ZIZHOU TANG
}

\begin{abstract}
AвsTRact. In this paper, two sequences of minimal isoparametric hypersurfaces are constructed via representations of Clifford algebras. Based on these, we give estimates on eigenvalues of the Laplacian of the focal submanifolds of isoparametric hypersurfaces in unit spheres. This improves results of [TY13] and [TXY14].

Eells and Lemaire [EL83] posed a problem to characterize the compact Riemannian manifold $M$ for which there is an eigenmap from $M$ to $S^{n}$. As another application of our constructions, the focal maps give rise to many examples of eigenmaps from minimal isoparametric hypersurfaces to unit spheres.

Most importantly, by investigating the second fundamental forms of focal submanifolds of isoparametric hypersurfaces in unit spheres, we provide infinitely many counterexamples to two conjectures of Leung [Le91] (posed in 1991) on minimal submanifolds in unit spheres. Notice that these conjectures of Leung have been proved in the case that the normal connection is flat HV01.
\end{abstract}

\section{INTRODUCTION}

Let $N$ be a connected complete Riemannian manifold. A non-constant smooth function $f$ on $N$ is called transnormal, if there exists a smooth function $b: \mathbb{R} \rightarrow \mathbb{R}$ such that the gradient of $f$ satisfies $|\nabla f|^{2}=b(f)$. Moreover, if there exists another function $a: \mathbb{R} \rightarrow$ $\mathbb{R}$ so that the Laplacian of $f$ satisfies $\Delta f=a(f)$, then $f$ is said to be isoparametric. Each regular level hypersurface of $f$ is then called an isoparametric hypersurface. It was proved by Wang (see [Wa87]) that each singular level set is also a smooth submanifold ( not necessarily connected ), the so-called focal submanifold. The whole family of isoparametric hypersurfaces together with the focal submanifolds form a singular Riemannian foliation, which is called the isoparametric foliation. For recent study of isoparametric functions on general Riemannian manifolds, especially on exotic spheres, see [GT13] and [QT15].

E. Cartan was the first to give a systematic study on isoparametric hypersurfaces in real space forms and proved that an isoparametric hypersurface is exactly a hypersurface with constant principal curvatures in these cases. For the spherical case (the most interesting and complicated case), Cartan obtained the classification result under the assumption that the number

2010 Mathematics Subject Classification. 53C42, 53C43, 53C99.

Key words and phrases. isoparametric foliation, Clifford algebra, harmonic map, Leung's conjecture.

The first author was partially supported by NSFC (No. 11401560, No. 11571339), and the second author(corresponding author) was partially supported by NSFC (No. 11331002). 
of the distinct principal curvatures is at most 3. Later, H. F. Münzner [Mü80] extended widely Cartan's work. To be precise, given an isoparametric hypersurface $M^{n}$ in $S^{n+1}(1)$, let $\xi$ be a unit normal vector field along $M^{n}$ in $S^{n+1}(1), g$ the number of distinct principal curvatures of $M, \cot \theta_{\alpha}\left(\alpha=1, \ldots, g ; 0<\theta_{1}<\cdots<\theta_{g}<\pi\right)$ the principal curvatures with respect to $\xi$ and $m_{\alpha}$ the multiplicity of $\cot \theta_{\alpha}$. Münzner proved that $m_{\alpha}=m_{\alpha+2}$ (indices $\bmod g$ ), $\theta_{\alpha}=\theta_{1}+\frac{\alpha-1}{g} \pi$ $(\alpha=1, \ldots, g)$, and there exists a homogeneous polynomial $F: \mathbb{R}^{n+2} \rightarrow \mathbb{R}$ of degree $g$, the so-called Cartan-Münzner polynomial, satisfying

$$
\left\{\begin{array}{l}
|\tilde{\nabla} F|^{2}=g^{2} r^{2 g-2}, \\
\tilde{\triangle} F=\frac{m_{2}-m_{1}}{2} g^{2} r^{g-2},
\end{array}\right.
$$

where $r=|x|, m_{1}$ and $m_{2}$ are the two multiplicities, and $\tilde{\nabla}, \tilde{\Delta}$ are Euclidean gradient and Laplacian, respectively. Moreover, Münzner obtained the remarkable result that $g$ must be $1,2,3,4$ or 6 . Since then, the classification of isoparametric hypersurfaces with $g=4$ or 6 in a unit sphere has been one of the most challenging problems in differential geometry.

Recently, due to [CCJ07], [Im08], [Ch11] and [Ch13], an isoparametric hypersurface with $g=4$ in a unit sphere must be homogeneous or OT-FKM type(see below) except for the case $\left(m_{1}, m_{2}\right)=(7,8)$. For $g=6$, R. Miyaoka [Mi13], [Mi16] completed the classification by showing that isoparametric hypersurfaces in this case are always homogeneous.

To prepare for our results, let us now recall the isoparametric hypersurfaces of OT-FKM type(c.f. [FKM81]). Given a symmetric Clifford system $\left\{P_{0}, \cdots, P_{m}\right\}$ on $\mathbb{R}^{2 l}$, i.e., $P_{0}, \ldots, P_{m}$ are symmetric matrices satisfying $P_{\alpha} P_{\beta}+P_{\beta} P_{\alpha}=2 \delta_{\alpha \beta} I_{2 l}$, Ferus, Karcher and Münzner defined a polynomial $F: \mathbb{R}^{2 l} \rightarrow \mathbb{R}$ by $F(x)=|x|^{4}-2 \sum_{\alpha=0}^{m}\left\langle P_{\alpha} x, x\right\rangle^{2}$. They verified that $f=\left.F\right|_{S^{2 l-1}(1)}$ is an isoparametric function on $S^{2 l-1}(1)$ and each level hypersurface of $f$ has 4 distinct constant principal curvatures with $\left(m_{1}, m_{2}\right)=(m, l-m-1)$, provided $m>0$ and $l-m-1>0$, where $l=k \delta(m)(k=1,2,3, \ldots)$ and $\delta(m)$ is the dimension of an irreducible module of the Clifford algebra $C_{m-1}$. As usual, for OT-FKM type, we denote the two focal submanifolds by $M_{+}=f^{-1}(1)$ and $M_{-}=f^{-1}(-1)$, which have codimension $m_{1}+1$ and $m_{2}+1$ in $S^{2 l-1}(1)$, respectively.

In the first part of the paper, inspired by the OT-FKM construction, for a symmetric Clifford system $\left\{P_{0}, \cdots, P_{m}\right\}$ on $\mathbb{R}^{2 l}$ with the Euclidean metric $\langle\cdot, \cdot\rangle$, we define $M_{i}:=\{x \in$ $\left.S^{2 l-1}(1) \mid\left\langle P_{0} x, x\right\rangle=\left\langle P_{1} x, x\right\rangle=\cdots=\left\langle P_{i} x, x\right\rangle=0\right\}$, and then we have a sequence

$$
M_{m}=M_{+} \subset M_{m-1} \subset \cdots \subset M_{0} \subset S^{2 l-1}(1) .
$$

For $0 \leq i \leq m-1$, it is natural to define a function $f_{i}: M_{i} \rightarrow \mathbb{R}$ by $f_{i}(x)=\left\langle P_{i+1} x, x\right\rangle$ for $x \in M_{i}$ (see also [TY12']).

Similarly, by defining $N_{i}:=\left\{x \in S^{2 l-1}(1) \mid\left\langle P_{0} x, x\right\rangle^{2}+\left\langle P_{1} x, x\right\rangle^{2}+\cdots+\left\langle P_{i} x, x\right\rangle^{2}=1\right\}$, we construct another sequence

$$
N_{1} \subset N_{2} \subset \cdots \subset N_{m}=M_{-} \subset S^{2 l-1}(1) .
$$


And for $2 \leq i \leq m$, we define a function $g_{i}: N_{i} \rightarrow \mathbb{R}$ by $g_{i}(x)=\left\langle P_{i} x, x\right\rangle$ for $x \in N_{i}$. Henceforth, we always regard $M_{i}$ and $N_{i}$ as Riemannian manifolds with the induced metric in $S^{2 l-1}(1)$. One of the main results in this paper is now stated as the following theorem.

Theorem 1.1. Assume the notations as above.

(1). For $0 \leq i \leq m-1$, the function $f_{i}: M_{i} \rightarrow \mathbb{R}$ with $\operatorname{Im}\left(f_{i}\right)=[-1,1]$ is an isoparametric function satisfying

$$
\left|\nabla f_{i}\right|^{2}=4\left(1-f_{i}^{2}\right), \Delta f_{i}=-4(l-i-1) f_{i} .
$$

For any $c \in(-1,1)$, the regular level set $\mathcal{U}_{c}=f_{i}^{-1}(c)$ has 3 distinct principal curvatures $-\sqrt{\frac{1-c}{1+c}}, 0, \sqrt{\frac{1+c}{1-c}}$ with multiplicities $l-i-2, i+1$, and $l-i-2$ respectively, w.r.t. the unit normal $\xi=\frac{\nabla f_{i}}{\left|\nabla f_{i}\right|}$. For $c= \pm 1$, the two focal submanifolds $\mathcal{U}_{ \pm 1}=f_{i}^{-1}( \pm 1)$ are both isometric to $S^{l-1}(1)$ and are totally geodesic in $M_{i}$.

Particularly, we have a minimal isoparametric sequence $M_{m} \subset M_{m-1} \subset \cdots \subset M_{0} \subset$ $S^{2 l-1}(1)$, i.e., each $M_{i+1}$ is a minimal isoparametric hypersurface in $M_{i}$ for $0 \leq i \leq m-1$. Moreover, $M_{i+j}$ is minimal in $M_{i}$.

(2). Similarly, for $2 \leq i \leq m$, the function $g_{i}: N_{i} \rightarrow \mathbb{R}$ with $\operatorname{Im}\left(g_{i}\right)=[-1,1]$ is an isoparametric function satisfying

$$
\left|\nabla g_{i}\right|^{2}=4\left(1-g_{i}^{2}\right), \Delta g_{i}=-4 i g_{i} .
$$

For any $c \in(-1,1)$, the regular level set $\mathcal{V}_{c}=g_{i}^{-1}(c)$ has 3 distinct principal curvatures $-\sqrt{\frac{1-c}{1+c}}, 0, \sqrt{\frac{1+c}{1-c}}$ with multiplicities $i-1, l-i$, and $i-1$ respectively, w.r.t. the unit normal $\eta=\frac{\nabla g_{i}}{\nabla g_{i} \mid}$. For $c= \pm 1$, the two focal submanifolds $\mathcal{V}_{ \pm 1}=g_{i}^{-1}( \pm 1)$ are both isometric to $S^{l-1}(1)$ and are totally geodesic in $N_{i}$.

In particular, we get another minimal isoparametric sequence $N_{1} \subset N_{2} \subset \cdots \subset N_{m} \subset$ $S^{2 l-1}(1)$, i.e., each $N_{i-1}$ is a minimal isoparametric hypersurface in $N_{i}$ for $2 \leq i \leq m$. Moreover, $N_{i}$ is minimal in $N_{i+j}$.

As a consequence, we have

Corollary 1.1. Assume the notations as in Theorem 1.1

(1). For $0 \leq i \leq m-1$, each $M_{i+1}$ fibers over $S^{l-1}$ with fiber $S^{l-i-2}$.

(2). For $2 \leq i \leq m$, each $N_{i-1}$ fibers over $S^{l-1}$ with fiber $S^{i-1}$.

Remark 1.1. For $i=m-1$, the first part of the above corollary gives a geometric interpretation of Lemma 3 in [Wa88].

Remark 1.2. Very recently, using representations of Clifford algebras, M. Radeschi in [Ra14] constructed indecomposable singular Riemannian foliations on round spheres, most of which are non-homogeneous. 
In next part, we will apply the above constructions of isoparametric functions to the estimates of eigenvalues. Given an $n$-dimensional closed Riemannian manifold $M^{n}$, recall that the Laplace-Beltrami operator acting on smooth functions on $M$ is an elliptic operator and has a discrete spectrum $\left\{0=\lambda_{0}(M)<\lambda_{1}(M) \leq \lambda_{2}(M) \leq \cdots \leq \lambda_{k}(M) \leq \cdots, k \uparrow \infty\right\}$, with each eigenvalue counted with its multiplicity. Following the way in [TY13] and [TXY14], we acquire the following theorem on eigenvalue estimates, based on isoparametric foliations constructed in Theorem 1.1

Theorem 1.2. Let $\left\{P_{0}, \cdots, P_{m}\right\}$ be a symmetric Clifford system on $\mathbb{R}^{2 l}$.

(1). For the sequence $M_{m} \subset M_{m-1} \subset \cdots \subset M_{0} \subset S^{2 l-1}(1)$, the following inequalities hold

a). $\lambda_{k}\left(M_{i}\right) \leq \frac{l-i-2}{l-i-3} \lambda_{k}\left(M_{i+1}\right)$ provided that $0 \leq i \leq m-1$ and $l-i-3>0$;

b). $\lambda_{k}\left(M_{i+1}\right) \leq 2 \lambda_{k}\left(S^{l-1}(1)\right)$ provided that $0 \leq i \leq m-1$.

(2). For the sequence $N_{1} \subset N_{2} \subset \cdots \subset N_{m} \subset S^{2 l-1}$ (1), the following inequalities hold

a). $\lambda_{k}\left(N_{i}\right) \leq \frac{i-1}{i-2} \lambda_{k}\left(N_{i-1}\right)$ provided that $3 \leq i \leq m$;

b). $\lambda_{k}\left(N_{i-1}\right) \leq 2 \lambda_{k}\left(S^{l-1}(1)\right)$ provided that $2 \leq i \leq m$.

In the third part, as an unexpected phenomenon, we find the relations between the focal maps of isoparametric foliations constructed in Theorem 1.1 and harmonic maps. To be more precise, let $M$ and $N$ be closed Riemannian manifolds, and $f$ a smooth map from $M$ to $N$. The energy functional $E(f)$ is defined by $E(f)=\frac{1}{2} \int_{M}|d f|^{2} d V_{M}$. The map $f$ is called harmonic if it is a critical point of the energy functional $E$. We refer to [EL78] and [EL88] for the background and development of this topic. For $N=S^{n}(1)$, a map $\varphi: M \rightarrow S^{n}(1)$ is called an eigenmap if the $\mathbb{R}^{n+1}$-components are eigenfunctions of the Laplacian of $M$ and all have the same eigenvalue. In particular, $\varphi$ is a harmonic map. In 1980, Eells and Lemaire ( See p. 70 of [EL83] ) posed the following

Problem 1.3. Characterize those compact $M$ for which there is an eigenmap $\varphi: M \rightarrow S^{n}(1)$ with $\operatorname{dim}(M) \geq n$ ?

In 1993, Eells and Ratto ( See p. 132 of [ER93] ) emphasized again that it is quite natural to study the eigenmaps to $S^{n}(1)$. As another application of our constructions in Theorem 1.1 we prove that

Theorem 1.4. Let $\left\{P_{0}, \cdots, P_{m}\right\}$ be a symmetric Clifford system on $\mathbb{R}^{2 l}$.

(1). For $0 \leq i \leq m-1$, both of the focal maps $\phi_{ \pm \frac{\pi}{4}}: M_{i+1} \rightarrow \mathcal{U}_{ \pm 1} \cong S^{l-1}(1)$ defined by

$$
\phi_{ \pm \frac{\pi}{4}}(x)=\frac{1}{\sqrt{2}}\left(x \pm P_{i+1} x\right), x \in M_{i+1},
$$

are submersive eigenmaps with the same eigenvalue $2 l-i-3$.

(2). For $2 \leq i \leq m$, both of the focal maps $\psi_{ \pm \frac{\pi}{4}}: N_{i-1} \rightarrow \mathcal{V}_{ \pm 1} \cong S^{l-1}(1)$ defined by

$$
\psi_{ \pm \frac{\pi}{4}}(x)=\frac{1}{\sqrt{2}}\left(x \pm P_{i} x\right), x \in N_{i}
$$


are submersive eigenmaps with the same eigenvalue $l+i-2$.

Meanwhile, the case for isoparametric foliations on unit spheres is also considered. Given an isoparametric hypersurface $M^{n}$ (not necessarily minimal) in $S^{n+1}(1)$ and a smooth field $\xi$ of unit normals to $M$, for each $x \in M$ and $\theta \in \mathbb{R}$, one has a map $\varphi_{\theta}: M^{n} \rightarrow S^{n+1}(1)$ by

$$
\varphi_{\theta}(x)=\cos \theta x+\sin \theta \xi(x) .
$$

If $\theta \neq \theta_{\alpha}$ for any $\alpha=1, \ldots, g, \varphi_{\theta}$ is a parallel hypersurface to $M$. If $\theta=\theta_{\alpha}$ for some $\alpha=$ $1, \ldots, g$, i.e., $\cot \theta=\cot \theta_{\alpha}$ is a principal curvature of $M, \varphi_{\theta}$ is not an immersion, actually a focal submanifold of codimension $m_{\alpha}+1$ in $S^{n+1}(1)$. And the map $\varphi_{\theta}$ from $M$ to a focal submanifold is said to be a focal map. Münzner [Mü80] asserted there are only two distinct focal submanifolds, and every isoparametric hypersurface is a tube of constant radius over each focal submanifold. Denote by $M_{+}$and $M_{-}$the focal submanifolds in $S^{n+1}(1)$ with codimension $m_{1}+1$ and $m_{2}+1$, respectively. However, there are more than two focal maps.

Proposition 1.1. Let $M$ be a closed isoparametric hypersurface in a unit sphere. Then every focal map from $M$ to its focal submanifolds $M_{+}$or $M_{-}$is harmonic.

Furthermore, we will investigate the stability of harmonic maps constructed in Theorem 1.4 and Proposition 1.1 as well.

The last part of the paper will be concerned with the pinching problem for minimal submanifolds in unit spheres. Let $W^{n}$ be a closed Riemannian manifold minimally immersed in $S^{n+p}(1)$. Let $B$ be the second fundamental form and define an extrinsic quantity $\sigma(W)=$ $\max \left\{|B(X, X)|^{2}|X \in T M| X \mid,=1\right\}$.

In 1986, H. Gauchman [Ga86] established a well known rigidity theorem which states that if $\sigma(W)<1 / 3$, then the submanifold $W$ must be totally geodesic. When the dimension $n$ of $W$ is even, the rigidity theorem above is optimal. As presented in [Ga86], there exist minimal submanifolds in unit spheres which are not totally geodesic, with $|B(X, X)|^{2} \equiv 1 / 3$ for any unit tangent vector $X$. When the dimension $n$ of $W$ is odd and $p>1$, the conclusion still holds under a weaker assumption $\sigma(W) \leq \frac{1}{3-2 / n}$. It is remarkable that Gauchman's rigidity theorem has been generalized to the case of submanifolds with parallel mean curvature in [XFX06].

In 1991, P. F. Leung [Le91] proved that if $n$ is odd, a closed minimally immersed submanifold $W^{n}$ with $\sigma(W) \leq \frac{n}{n-1}$ is totally geodesic provided that the normal connection is flat. Based on this fact, he proposed the following

Conjecture 1.5. If $n$ is odd, $W^{n}$ is minimally immersed in $S^{n+p}(1)$ with $\sigma(W) \leq \frac{n}{n-1}$, then $W$ is homeomorphic to $S^{n}$.

By investigating the second fundamental form of the Clifford minimal hypersurfaces in unit spheres, Leung also posed the following stronger 
Conjecture 1.6. If $n$ is odd and $W^{n}$ is minimally immersed in $S^{n+p}(1)$ with $\sigma(W)<\frac{n+1}{n-1}$, then $W$ is homeomorphic to $S^{n}$.

For minimal submanifolds in unit spheres with flat normal connections, Conjecture 1.6 was proved by T. Hasanis and T. Vlachos [HV01]. In fact, they showed that the condition $\operatorname{Ric}(W)>\frac{n(n-3)}{n-1}$ is equivalent to the inequality $\sigma(W)<\frac{n+1}{n-1}$. Thus in the case that the normal connection is flat, Conjecture 1.6 follows from Theorem B in [HV01].

Recall that the examples with even dimensions and $\sigma(W)=1 / 3$ given in [Ga86] originated from the Veronese embeddings of the projective planes $\mathbb{R} P^{2}, \mathbb{C} P^{2}, \mathbb{H} P^{2}$ and $\mathbb{O} P^{2}$ in $S^{4}(1)$, $S^{7}(1), S^{13}(1)$ and $S^{25}(1)$, respectively. Observe that those Veronese submanifolds are just the focal submanifolds of isoparametric hypersurfaces in unit spheres with $g=3$. Hence, it is very natural for us to consider the case with $g=4$.

Theorem 1.7. Let $M^{n}$ be an isoparametric hypersurface in $S^{n+1}(1)$ with $g=4$ and multiplicities $\left(m_{1}, m_{2}\right)$, and denote by $M_{+}$and $M_{-}$the focal submanifolds of $M^{n}$ in $S^{n+1}(1)$ with dimension $m_{1}+2 m_{2}$ and $2 m_{1}+m_{2}$ respectively. Then $M_{ \pm}$are minimal in $S^{n+1}(1)$ with $\sigma\left(M_{ \pm}\right)=1$. However, $M_{ \pm}$are not homeomorphic to the spheres.

Remark 1.3. 1). If $m_{1}$ is odd, $M_{+} \subset S^{n+1}(1)$ in Theorem 1.7 is a counterexample to Conjecture 1.5 and Conjecture 1.6 Similarly, if $m_{2}$ is odd, $M_{-} \subset S^{n+1}(1)$ can be also served as a counterexample to both of the conjectures.

2). It is not difficult to show directly that the normal connections of those focal submanifolds in unit spheres are non-flat despite the dimensions.

The present paper is organized as follows. In Section 2, we will prove Theorem 1.1 and give a detailed investigation into the geometric properties of isoparametric foliations we constructed. Based on Section 2, Theorem 1.2 will be proved in Section 3. In Section 4, we are mainly concerned with the harmonicity of the focal maps. Moreover, the stability will be studied as well. Finally in Section 5, infinitely many counterexamples will be provided to the conjectures of Leung.

\section{CONSTRuctions OF ISOPARAMETRIC FOLIATIONS}

The aim of this section is to give a complete proof of Theorem 1.1. For convenience, the proof will be divided into several lemmas.

Lemma 2.1. Let $\left\{P_{0}, \cdots, P_{m}\right\}$ be a symmetric Clifford system on $\mathbb{R}^{2 l}$ and $M_{i}, N_{i}$ defined in the introduction. For $0 \leq i \leq m-1$, the function $f_{i}: M_{i} \rightarrow \mathbb{R}, x \mapsto\left\langle P_{i+1} x, x\right\rangle$, is an isoparametric function with $\operatorname{Im}\left(f_{i}\right)=[-1,1]$ and satisfies

$$
\left|\nabla f_{i}\right|^{2}=4\left(1-f_{i}^{2}\right), \Delta f_{i}=-4(l-i-1) f_{i} .
$$


While for $2 \leq i \leq m$, the function $g_{i}: N_{i} \rightarrow \mathbb{R}, x \mapsto\left\langle P_{i} x, x\right\rangle$, is also an isoparametric function with $\operatorname{Im}\left(g_{i}\right)=[-1,1]$ and satisfies

$$
\left|\nabla g_{i}\right|^{2}=4\left(1-g_{i}^{2}\right), \Delta g_{i}=-4 i g_{i} .
$$

Proof. First, the function $f_{i}$ on $M_{i}$ will be considered. According to [FKM81],$M_{i}$ is a smooth submanifold in $S^{2 l-1}(1) \subset \mathbb{R}^{2 l}$ with $\operatorname{dim} M_{i}=2 l-i-2$. As we defined in the introduction, it is convenient to regard the function $f_{i}$ as the restriction of the function $F_{i}: \mathbb{R}^{2 l} \rightarrow \mathbb{R}, x \mapsto$ $\left\langle P_{i+1} x, x\right\rangle$ to $M_{i}$. Henceforth, we will denote the covariant derivatives and Laplacians of $M_{i}$ and $\mathbb{R}^{2 l}$ by $\nabla, \triangle$ and $\tilde{\nabla}, \tilde{\Delta}$ respectively. For any $x \in M_{i}, v_{x} M_{i}$, the normal space of $M_{i}$ in $\mathbb{R}^{2 l}$ at $x$, is equal to $\operatorname{Span}\left\{P_{0} x, \cdots, P_{i} x, x\right\}$. By a direct computation and the property of the symmetric Clifford system, we have $\tilde{\nabla} F_{i}=2 P_{i+1} x$, and $\left\langle\tilde{\nabla} F_{i}, P_{\alpha} x\right\rangle=0$ for any $0 \leq \alpha \leq i$. And it follows that

$$
\begin{aligned}
\nabla f_{i} & =\tilde{\nabla} F_{i}-\left\langle\tilde{\nabla} F_{i}, x\right\rangle x-\sum_{\alpha=0}^{i}\left\langle\tilde{\nabla} F_{i}, P_{\alpha} x\right\rangle P_{\alpha} x \\
& =\tilde{\nabla} F_{i}-\left\langle\tilde{\nabla} F_{i}, x\right\rangle x \\
& =2 P_{i+1} x-2\left\langle P_{i+1} x, x\right\rangle x .
\end{aligned}
$$

Hence $\left|\nabla f_{i}\right|^{2}=4\left(1-f_{i}^{2}\right)$. For the Laplacian of $f_{i}$, we note that

$$
\text { Hess } f_{i}(X, Y)=\widetilde{\operatorname{Hess}} F_{i}(X, Y)+B(X, Y)\left(F_{i}\right)
$$

for $X, Y \in T_{x} M_{i}$, where $B$ is the second fundamental form of $M_{i}$ in $\mathbb{R}^{2 l}$, and Hess $f_{i}, \widetilde{\operatorname{Hess}} F_{i}$ are the Hessians of $f_{i}, F_{i}$, respectively. Choose an orthonormal basis $\left\{e_{a}\right\}_{a=1}^{2 l-i-2}$ for $T_{x} M_{i}$. In virtue of Münzner [Mü80], $M_{i}$ is minimal in $S^{2 l-1}(1)$, i.e., $\sum_{a}\left\langle B\left(e_{a}, e_{a}\right), P_{\alpha} x\right\rangle=0$, for $0 \leq \alpha \leq i$. Thus,

$$
\begin{aligned}
\Delta f_{i} & =\sum_{a} \operatorname{\operatorname {Hess}} f_{i}\left(e_{a}, e_{a}\right) \\
& =\sum_{a} \widetilde{\operatorname{Hess}} F_{i}\left(e_{a}, e_{a}\right)+\sum_{a} B\left(e_{a}, e_{a}\right)\left(F_{i}\right) \\
& =\sum_{a} \widetilde{\operatorname{Hess}} F_{i}\left(e_{a}, e_{a}\right)+\left\langle\sum_{a} B\left(e_{a}, e_{a}\right), x\right\rangle x\left(F_{i}\right) \\
& =\sum_{a} \widetilde{\operatorname{Hess}} F_{i}\left(e_{a}, e_{a}\right)-2(2 l-i-2) f_{i},
\end{aligned}
$$

where we have used $\left\langle\sum_{a} B\left(e_{a}, e_{a}\right), x\right\rangle=-(2 l-i-2)$ and $x\left(F_{i}\right)=2 f_{i}$. Moreover,

$$
\begin{aligned}
\sum_{a} \widetilde{\operatorname{Hess}} F_{i}\left(e_{a}, e_{a}\right) & =\tilde{\Delta} F_{i}-\sum_{\alpha=0}^{i} \widetilde{\operatorname{Hess}} F_{i}\left(P_{\alpha} x, P_{\alpha} x\right)-\widetilde{\operatorname{Hess}} F_{i}(x, x) \\
& =\operatorname{Tr} P_{i+1}+2(i+1) f_{i}-2 f_{i} \\
& =2 i f_{i}
\end{aligned}
$$

where $\tilde{\Delta} F_{i}=\operatorname{Tr} P_{i+1}=0$ and $\widetilde{\operatorname{Hess}} F_{i}\left(P_{\alpha} x, P_{\alpha} x\right)=-2 f_{i}$ by using the properties of symmetric Clifford system. In conclusion, $\triangle f_{i}=-4(l-i-1) f_{i}$. 
Next, we will deal with the function $g_{i}$ on $N_{i}$. According to [FKM81],$N_{i}$ is also a smooth submanifold in $S^{2 l-1}(1) \subset \mathbb{R}^{2 l}$ with $\operatorname{dim} N_{i}=l+i-1$. It is also convenient to regard the function $g_{i}$ as the restriction of the function $G_{i}: \mathbb{R}^{2 l} \rightarrow \mathbb{R}, x \mapsto\left\langle P_{i} x, x\right\rangle$ to $N_{i}$. With no possibility of confusion, we will also denote the covariant derivative and Laplacian of $N_{i}$ by $\nabla$ and $\Delta$, respectively. For any $x \in N_{i}$, we can define $\mathcal{P}=\sum_{\alpha=0}^{i}\left\langle P_{\alpha} x, x\right\rangle P_{\alpha}$. And thus $\mathcal{P}_{x}=x$. According to [FKM81], the normal space of $N_{i}$ in $\mathbb{R}^{2 l}$ at $x$, denoted by $v_{x} N_{i}$, is equal to

$$
\left\{\varsigma \in E_{-}(\mathcal{P}) \mid \varsigma \perp Q x, \forall Q \in \Sigma\left(P_{0}, \cdots, P_{i}\right),\langle Q, \mathcal{P}\rangle=0\right\} \oplus \mathbb{R} x,
$$

where $E_{-}(\mathcal{P})$ is eigenspace of $\mathcal{P}$ for the eigenvalue -1 and $\Sigma\left(P_{0}, \cdots, P_{i}\right)$ the Clifford sphere spanned by $P_{0}, \cdots, P_{i}$. Since $\tilde{\nabla} G_{i}=2 P_{i} x$ and $\langle Q, \mathcal{P}\rangle=0$,

$$
\nabla g_{i}=2 P_{i} x-2\left\langle P_{i} x, x\right\rangle x=2\left(P_{i}-\left\langle P_{i} x, x\right\rangle \mathcal{P}\right) x=2 Q x,
$$

where $Q=P_{i}-\left\langle P_{i} x, x\right\rangle \mathcal{P}$ (also see [TY12]). As a result, $\left|\nabla g_{i}\right|^{2}=4\left(1-g_{i}^{2}\right)$. At last, due to [So92], the equation of Laplacian of $g_{i}$ holds.

Furthermore, the following lemma investigates the extrinsic geometry of isoparametric hypersurfaces given by the preceding lemma.

Lemma 2.2. (1). For the isoparametric function $f_{i}$ on $M_{i}$ and any $c \in(-1,1)$, the regular level set $\mathcal{U}_{c}=f_{i}^{-1}(c)$ has 3 distinct constant principal curvatures $-\sqrt{\frac{1-c}{1+c}}, 0, \sqrt{\frac{1+c}{1-c}}$ with multiplicities $l-i-2, i+1$, and $l-i-2$ respectively, w.r.t. the unit normal $\xi=\frac{\nabla f_{i}}{\left|\nabla f_{i}\right|}$. Moreover, for any $x \in \mathcal{U}_{c}$, the corresponding principal spaces are

$$
\begin{aligned}
T_{-\sqrt{\frac{1-c}{1+c}}(\xi)} & =E_{+}\left(P_{i+1}\right) \cap T_{x} \mathcal{U}_{c}, \\
T_{\sqrt{\frac{1+c}{1-c}}} & =E_{-}\left(P_{i+1}\right) \cap T_{x} \mathcal{U}_{c}, \\
T_{0}(\xi) & =\left\{Q \xi \mid Q \in \mathbb{R} \Sigma\left(P_{0}, \cdots, P_{i}\right)\right\}
\end{aligned}
$$

where $E_{ \pm}\left(P_{i+1}\right)$ are eigenspaces of $P_{i+1}$ for the eigenvalues \pm 1 with $\operatorname{dim} E_{ \pm}\left(P_{i+1}\right)=l$, and $\Sigma\left(P_{0}, \cdots, P_{i}\right)$ is the Clifford sphere spanned by $P_{0}, \cdots, P_{i}$.

(2). For the isoparametric function $g_{i}$ on $N_{i}$ and any $c \in(-1,1)$, the regular level set $\mathcal{V}_{c}=g_{i}^{-1}(c)$ has 3 distinct constant principal curvatures $-\sqrt{\frac{1-c}{1+c}}, \sqrt{\frac{1+c}{1-c}}, 0$, with multiplicities $i-1, i-1$ and $l-i$, respectively, w.r.t. the unit normal $\eta=\frac{\nabla g_{i}}{\left|\nabla g_{i}\right|}$. Moreover, for any $x \in \mathcal{V}_{c}$, the corresponding principal spaces are

$$
\begin{aligned}
T_{-\sqrt{\frac{1-c}{1+c}}}(\eta) & =E_{+}\left(P_{i}\right) \cap T_{x} \mathcal{V}_{c}=\operatorname{Span}\left\{Q\left(x-P_{i} x\right) \mid Q \in \Sigma\left(P_{0}, \cdots, P_{i-1}\right),\langle Q, Q\rangle=0\right\}, \\
T_{\sqrt{\frac{1+c}{1-c}}} & =E_{-}\left(P_{i}\right) \cap T_{x} \mathcal{V}_{c}=\operatorname{Span}\left\{Q\left(x+P_{i} x\right) \mid Q \in \Sigma\left(P_{0}, \cdots, P_{i-1}\right),\langle Q, Q\rangle=0\right\}, \\
T_{0}(\eta) & =\left\{X \in E_{+}(\mathcal{P}) \mid\langle X, x\rangle=0,\left\langle X, P_{i} R x\right\rangle=0, \forall R \in \Sigma\left(P_{0}, \cdots, P_{i}\right),\langle R, \mathcal{P}\rangle=0\right\},
\end{aligned}
$$

where $\mathcal{P}=\sum_{\alpha=0}^{i}\left\langle P_{\alpha} x, x\right\rangle P_{\alpha}$, and $\mathcal{Q}=\frac{1}{\sqrt{1-c^{2}}}\left(\left\langle P_{0} x, x\right\rangle P_{0}+\cdots+\left\langle P_{i-1} x, x\right\rangle P_{i-1}\right)$. 
Proof. (1). For any $c \in(-1,1)$, it follows from Lemma 2.1 that $\mathcal{U}_{c}$ is an isoparametric hypersurface in $M_{i}$ with $\operatorname{dim} \mathcal{U}_{c}=2 l-i-3$, and for each $x \in \mathcal{U}_{c} \subset M_{i}$, the unit normal $\xi=\frac{1}{\sqrt{1-c^{2}}}\left(P_{i+1} x-c x\right)$. Hence, the corresponding shape operator $A_{\xi}: T_{x} \mathcal{U}_{c} \rightarrow T_{x} \mathcal{U}_{c}$ is given by $A_{\xi} X=-\frac{1}{\sqrt{1-c^{2}}}\left(\left(P_{i+1} X\right)^{T}-c X\right)$, for each $X \in T_{x} \mathcal{U}_{c}$, where $\left(P_{i+1} X\right)^{T}$ is the tangential component of $P_{i+1} X$ in $T_{x} \mathcal{U}_{c}$.

Suppose $X \in E_{+}\left(P_{i+1}\right) \cap T_{x} \mathcal{U}_{c}$. Then $A_{\xi} X=-\sqrt{\frac{1-c}{1+c}} X$ and $X \in T_{-\sqrt{\frac{1-c}{1+c}}}(\xi)$. Hence, $E_{+}\left(P_{i+1}\right) \cap T_{x} \mathcal{U}_{c} \subset T_{-\sqrt{\frac{1-c}{1+c}}}(\xi)$. Notice that

$$
\begin{aligned}
E_{+}\left(P_{i+1}\right) \cap T_{x} \mathcal{U}_{c} & =\left\{X \in E_{+}\left(P_{i+1}\right) \mid\langle X, x\rangle=0,\langle X, \xi\rangle=0,\langle X, Q x\rangle=0, \forall Q \in \Sigma\left(P_{0}, \cdots, P_{i}\right)\right\} \\
& =\left\{X \in E_{+}\left(P_{i+1}\right) \mid\langle X, Q x\rangle=0, \forall Q \in \Sigma\left(P_{0}, \cdots, P_{i}, P_{i+1}\right)\right\}
\end{aligned}
$$

so $\operatorname{dim}\left(E_{+}\left(P_{i+1}\right) \cap T_{x} \mathcal{U}_{c}\right) \geq l-i-2$.

Next, suppose $X \in E_{-}\left(P_{i+1}\right) \cap T_{x} \mathcal{U}_{c}$. Then as above, we have $A_{\xi} X=\sqrt{\frac{1+c}{1-c}} X$ and $X \in$ $T_{\sqrt{\frac{1+c}{1-c}}}(\xi)$. Hence, $E_{-}\left(P_{i+1}\right) \cap T_{x} \mathcal{U}_{c} \subset T_{\sqrt{\frac{1+c}{1-c}}}(\xi)$ and

$$
E_{-}\left(P_{i+1}\right) \cap T_{x} \mathcal{U}_{c}=\left\{X \in E_{-}\left(P_{i+1}\right) \mid\langle X, Q x\rangle=0, \forall Q \in \Sigma\left(P_{0}, \cdots, P_{i}, P_{i+1}\right)\right\}
$$

which implies that $\operatorname{dim}\left(E_{-}\left(P_{i+1}\right) \cap T_{x} \mathcal{U}_{c}\right) \geq l-i-2$.

At last, suppose $X=Q \xi$ for $Q \in \Sigma\left(P_{0}, \cdots, P_{i}\right)$. We need to show that $X \in T_{x} \mathcal{U}_{c}$. Observe that

$$
\begin{aligned}
\langle X, x\rangle & =\langle\xi, Q x\rangle=\frac{1}{\sqrt{1-c^{2}}}\left\langle P_{i+1} x-c x, Q x\right\rangle=0, \\
\langle X, \xi\rangle & =\langle Q \xi, \xi\rangle=\frac{1}{1-c^{2}}\left\langle Q\left(P_{i+1} x-c x\right), P_{i+1} x-c x\right\rangle=0, \\
\langle X, Q x\rangle & =\langle\xi, x\rangle=\frac{1}{\sqrt{1-c^{2}}}\left\langle P_{i+1} x-c x, x\right\rangle=0 .
\end{aligned}
$$

Moreover, for $P \in \Sigma\left(P_{0}, \cdots, P_{i}\right)$ with $\langle P, Q\rangle=0$,

$$
\begin{aligned}
\langle X, P x\rangle & =\frac{1}{\sqrt{1-c^{2}}}\left\langle Q\left(P_{i+1} x-c x\right) P x\right\rangle, \\
& =\frac{1}{\sqrt{1-c^{2}}}\left\langle Q P_{i+1} x, P x\right\rangle-\frac{c}{\sqrt{1-c^{2}}}\langle Q x, P x\rangle \\
& =0,
\end{aligned}
$$


where the fact that $P Q P_{i+1}$ is skew-symmetric has been used. Thus $X \in T_{x} \mathcal{U}_{c}$. Now, $A_{\xi} X=$ $-\frac{1}{\sqrt{1-c^{2}}}\left(\left(P_{i+1} Q \xi\right)^{T}-c Q \xi\right)=0$, since

$$
\begin{aligned}
\left(P_{i+1} Q \xi\right)^{T} & =\frac{1}{\sqrt{1-c^{2}}}\left(P_{i+1} Q\left(P_{i+1} x-c x\right)\right)^{T} \\
& =\frac{1}{\sqrt{1-c^{2}}}\left(-Q x+c Q P_{i+1} x\right)^{T} \\
& =\frac{1}{\sqrt{1-c^{2}}}\left(c Q P_{i+1} x-c^{2} Q x\right)^{T} \\
& =c Q \xi
\end{aligned}
$$

So $\left\{Q \xi \mid Q \in \mathbb{R} \Sigma\left(P_{0}, \cdots, P_{i}\right)\right\} \subset T_{0}(\xi)$ and $\operatorname{dim}\left\{Q \xi \mid Q \in \mathbb{R} \Sigma\left(P_{0}, \cdots, P_{i}\right)\right\}=i+1$.

We have constructed three mutually orthogonal subspaces of $T_{x} \mathcal{U}_{c}$ and the sum of the dimensions is no less than $i+1+2(l-i-2)=\operatorname{dim} T_{x} \mathcal{U}_{c}$. Hence part (1) of the lemma follows.

(2). Analogous to part (1), for any $c \in(-1,1)$, it also follows from Lemma 2.1 that $\mathcal{V}_{c}$ is an isoparametric hypersurface in $N_{i}$ with $\operatorname{dim} \mathcal{V}_{c}=l+i-2$. For each $x \in \mathcal{V}_{c} \subset N_{i}$, the unit normal $\eta=\frac{1}{\sqrt{1-c^{2}}}\left(P_{i} x-c x\right)$ and the corresponding shape operator $A_{\eta}: T_{x} \mathcal{V}_{c} \rightarrow T_{x} \mathcal{V}_{c}$ is given by $A_{\eta} X=-\frac{1}{\sqrt{1-c^{2}}}\left(\left(P_{i} X\right)^{T}-c X\right)$, for each $X \in T_{x} \mathcal{V}_{c}$, where $\left(P_{i} X\right)^{T}$ is the tangential component of $P_{i} X$ in $T_{x} \mathcal{V}_{c}$.

Suppose $X=Q\left(x-P_{i} x\right)$ for $Q \in \Sigma\left(P_{0}, \cdots, P_{i-1}\right)$ with $\langle Q, Q\rangle=0$. Then

$$
P_{i} X=P_{i} Q\left(x-P_{i} x\right)=Q\left(-P_{i} x+x\right)=X,
$$

and $X \in E_{+}\left(P_{i}\right)$. The next task for us is to show $X \in T_{x} \mathcal{V}_{c}$. It follows from $\langle Q, Q\rangle=0$ that $\langle Q, \mathcal{P}\rangle=0$, and consequently,

$$
\begin{aligned}
\langle X, x\rangle & =\left\langle Q\left(x-P_{i} x\right), x\right\rangle \\
& =\langle Q x, x\rangle-\left\langle Q x, P_{i} x\right\rangle \\
& =\left\langle Q x, \mathcal{P}_{x}\right\rangle \\
& =0 .
\end{aligned}
$$

and

$$
\begin{aligned}
\langle X, \eta\rangle & =\frac{1}{\sqrt{1-c^{2}}}\left\langle Q\left(x-P_{i} x\right), P_{i} x-c x\right\rangle \\
& =\sqrt{\frac{1-c}{1+c}}\langle Q x, x\rangle \\
& =\sqrt{\frac{1-c}{1+c}}\langle Q x, \mathcal{P} x\rangle \\
& =0,
\end{aligned}
$$

where the equalities $\left\langle Q, P_{i}\right\rangle=0$ and $\mathcal{P}_{x}=x$ have been used. Since

$$
v_{x} N_{i}=\left\{\varsigma \in E_{-}(\mathcal{P}) \mid \varsigma \perp Q x, \forall Q \in \Sigma\left(P_{0}, \cdots, P_{i}\right),\langle Q, \mathcal{P}\rangle=0\right\} \oplus \mathbb{R} x
$$


as in Lemma 2.1, to prove $X \in T_{x} \mathcal{V}_{c}$, it is sufficient to show $\langle X, \zeta\rangle=0$ for each normal vector $\zeta \in\left\{\varsigma \in E_{-}(\mathcal{P}) \mid \varsigma \perp Q x, \forall Q \in \Sigma\left(P_{0}, \cdots, P_{i}\right),\langle Q, \mathcal{P}\rangle=0\right\}$. Actually, $\langle X, \zeta\rangle=$ $\langle Q x, \zeta\rangle-\left\langle Q P_{i} x, \zeta\right\rangle=-\left\langle Q P_{i} x, \zeta\right\rangle$. Furthermore, $\left\langle Q P_{i} x, \zeta\right\rangle=\left\langle\mathcal{P} Q P_{i} x, \mathcal{P} \zeta\right\rangle=-\left\langle\mathcal{P} Q P_{i} x, \zeta\right\rangle=$ $\left\langle Q \mathcal{P} P_{i} x, \zeta\right\rangle=\left\langle Q\left(-P_{i} \mathcal{P}+2\left\langle P_{i} x, x\right\rangle I_{2 l}\right) x, \zeta\right\rangle=-\left\langle Q P_{i} x, \zeta\right\rangle+2\left\langle P_{i} x, x\right\rangle\langle Q x, \zeta\rangle=-\left\langle Q P_{i} x, \zeta\right\rangle$, where the identity $\mathcal{P} P_{i}=-P_{i} \mathcal{P}+2\left\langle P_{i} x, x\right\rangle I_{2 l}$ has been used. It follows that $\langle X, \zeta\rangle=0$ and $X \in T_{x} \mathcal{V}_{c}$. Then $A_{\eta} X=-\sqrt{\frac{1-c}{1+c}} X$ and

$$
\operatorname{Span}\left\{Q\left(x-P_{i} x\right) \mid Q \in \Sigma\left(P_{0}, \cdots, P_{i-1}\right),\langle Q, Q\rangle=0\right\} \subset E_{+}\left(P_{i}\right) \cap T_{x} \mathcal{V}_{c} \subset T_{-\sqrt{\frac{1-c}{1+c}}}(\eta),
$$

with $\operatorname{dimSpan}\left\{Q\left(x-P_{i} x\right) \mid Q \in \Sigma\left(P_{0}, \cdots, P_{i-1}\right),\langle Q, Q\rangle=0\right\}=i-1$.

Similarly, suppose $X=Q\left(x+P_{i} x\right)$ for $Q \in \Sigma\left(P_{0}, \cdots, P_{i-1}\right)$ with $\langle Q, Q\rangle=0$. Then $X \in E_{-}\left(P_{i}\right) \cap T_{x} \mathcal{V}_{c}, A_{\eta} X=\sqrt{\frac{1+c}{1-c}} X$ and

$$
\operatorname{Span}\left\{Q\left(x+P_{i} x\right) \mid Q \in \Sigma\left(P_{0}, \cdots, P_{i-1}\right),\langle Q, Q\rangle=0\right\} \subset E_{-}\left(P_{i}\right) \cap T_{x} \mathcal{V}_{c} \subset T \sqrt{\sqrt{\frac{1+c}{1-c}}}(\eta),
$$

with $\operatorname{dim} \operatorname{Span}\left\{Q\left(x+P_{i} x\right) \mid Q \in \Sigma\left(P_{0}, \cdots, P_{i-1}\right),\langle Q, Q\rangle=0\right\}=i-1$.

Now, suppose $X \in E_{+}(\mathcal{P}),\langle X, x\rangle=0$, and $\left\langle X, P_{i} R x\right\rangle=0$, for arbitrary $R \in \Sigma\left(P_{0}, \cdots, P_{i}\right)$ with $\langle R, \mathcal{P}\rangle=0$. In this case, to prove $X \in T_{x} \mathcal{V}_{c}$, it is sufficient to verify $\langle X, \eta\rangle=0$. In fact, we have $\langle X, \eta\rangle=\frac{1}{\sqrt{1-c^{2}}}\left\langle X, P_{i} x-c x\right\rangle=\frac{1}{\sqrt{1-c^{2}}}\left\langle X, P_{i} x\right\rangle$ and

$$
\left\langle X, P_{i} x\right\rangle=\left\langle\mathcal{P} X, \mathcal{P} P_{i} x\right\rangle=\left\langle X,-P_{i} \mathcal{P}_{x}+2\left\langle P_{i} x, x\right\rangle x\right\rangle=-\left\langle X, P_{i} x\right\rangle,
$$

where the identity $\mathcal{P} P_{i}=-P_{i} \mathcal{P}+2\left\langle P_{i} x, x\right\rangle I_{2 l}$ has been used. Hence, $X \in T_{x} \mathcal{V}_{c}$. Then we will show that $A_{\eta} X=0$. Observe that in this case

$$
\begin{aligned}
A_{\eta} X=0 & \Leftrightarrow\left(P_{i} X\right)^{T}=c X \\
& \Leftrightarrow P_{i} X-c X \in\left\{\varsigma \in E_{-}(\mathcal{P}) \mid \varsigma \perp Q x, \forall Q \in \Sigma\left(P_{0}, \cdots, P_{i}\right),\langle Q, \mathcal{P}\rangle=0\right\} .
\end{aligned}
$$

It is sufficient to prove

$$
P_{i} X-c X \in\left\{\varsigma \in E_{-}(\mathcal{P}) \mid \varsigma \perp Q x, \forall Q \in \Sigma\left(P_{0}, \cdots, P_{i}\right),\langle Q, \mathcal{P}\rangle=0\right\} .
$$

First, it is not difficult to prove that

$$
\mathcal{P}\left(P_{i} X-c X\right)=-\left(P_{i} X-c X\right) \Leftrightarrow \mathcal{P} X=X,
$$

and therefore $P_{i} X-c X \in E_{-}(\mathcal{P})$. Next, for $Q \in \Sigma\left(P_{0}, \cdots, P_{i}\right)$ with $\langle Q, \mathcal{P}\rangle=0$, we have $\left\langle P_{i} X-c X, Q x\right\rangle=\left\langle X, P_{i} Q x\right\rangle-c\langle X, Q x\rangle$ and $\langle X, Q x\rangle=\langle\mathcal{P} X, \mathcal{P} Q x\rangle=-\langle X, Q \mathcal{P} x\rangle=-\langle X, Q x\rangle$. It follows that $\left\langle P_{i} X-c X, Q x\right\rangle=\left\langle X, P_{i} Q x\right\rangle=0$ by the definition of $X$. In a word, $A_{\eta} X=0$ and

$$
\left\{X \in E_{+}(\mathcal{P}) \mid\langle X, x\rangle=0,\left\langle X, P_{i} R x\right\rangle=0, \forall R \in \Sigma\left(P_{0}, \cdots, P_{i}\right),\langle R, \mathcal{P}\rangle=0\right\} \subset T_{0}(\eta) .
$$

Moreover, we claim that if $X \in E_{+}(\mathcal{P})$, and $\left\langle X, P_{i} R x\right\rangle=0$ for any $R \in \Sigma\left(P_{0}, \cdots, P_{i}\right)$ with $\langle R, \mathcal{P}\rangle=0$, then $\langle X, x\rangle=0$. To prove the claim, define $a_{0}=\left\langle P_{i}, \mathcal{P}\right\rangle$. Then $\left|a_{0}\right|<1$, and $P_{i}-a_{0} \mathcal{P} \in \Sigma\left(P_{0}, \cdots, P_{i}\right)$ with $\left\langle P_{i}-a_{0} \mathcal{P}, \mathcal{P}\right\rangle=0$. Hence, $\left\langle X, P_{i}\left(P_{i}-a_{0} \mathcal{P}\right) x\right\rangle=\langle X, x\rangle-$ $a_{0}\left\langle X, P_{i} x\right\rangle=0$. Observing that

$$
\left\langle X, P_{i} x\right\rangle=\left\langle\mathcal{P} X, \mathcal{P} P_{i} x\right\rangle=\left\langle X,\left(-P_{i} \mathcal{P}+2\left\langle P_{i} x, x\right\rangle I_{2 l}\right) x\right\rangle=-\left\langle X, P_{i} x\right\rangle+2\left\langle P_{i} x, x\right\rangle\langle X, x\rangle,
$$


we see $\left\langle X, P_{i} x\right\rangle=c\langle X, x\rangle$. And thus $\langle X, x\rangle-a_{0} c\langle X, x\rangle=0$. Since $\left|a_{0}\right|,|c|<1$, it follows $\langle X, x\rangle=0$. Due to the claim above, it follows that

$$
\operatorname{dim}\left\{X \in E_{+}(\mathcal{P}) \mid\langle X, x\rangle=0,\left\langle X, P_{i} R x\right\rangle=0, \forall R \in \Sigma\left(P_{0}, \cdots, P_{i}\right),\langle R, \mathcal{P}\rangle=0\right\} \geq l-i .
$$

Since three mutually orthogonal subspaces of $T_{x} \mathcal{V}_{c}$ are constructed and the sum of the dimensions is no less than $l-i+2(i-1)=\operatorname{dim} T_{x} \mathcal{V}_{c}$, part (2) of the lemma follows.

Lemma 2.3. For $c= \pm 1, \mathcal{U}_{ \pm 1}=E_{ \pm}\left(P_{i+1}\right) \cap S^{2 l-1}(1)$, denoted by $S E_{ \pm}\left(P_{i+1}\right)$, and the two focal submanifolds $\mathcal{U}_{ \pm 1}$ are both isometric to $S^{l-1}(1)$ and are totally geodesic in $M_{i}$. Similarly, $\mathcal{V}_{ \pm 1}=E_{ \pm}\left(P_{i}\right) \cap S^{2 l-1}(1)$, denoted by $S E_{ \pm}\left(P_{i}\right)$, and $\mathcal{V}_{ \pm 1}$ are both isometric to $S^{l-1}(1)$ and are totally geodesic in $N_{i}$.

Proof. Because $\mathcal{U}_{ \pm 1}=\left\{x \in S^{2 l-1}(1) \mid\left\langle P_{0} x, x\right\rangle=\cdots=\left\langle P_{i} x, x\right\rangle=0,\left\langle P_{i+1} x, x\right\rangle= \pm 1\right\}$ by definition, it follows from Cauchy-Schwarz inequality and the properties of the symmetric Clifford system that $\mathcal{U}_{ \pm 1}=\left\{x \in S^{2 l-1}(1) \mid P_{i+1} x= \pm x\right\}$. Thus, the first part of the lemma is proved. And an analogous argument implies the second part of the lemma.

We are now in the position to give a

\section{Proof of Theorem 1.1:}

Proof. In order to complete the proof, it remains to show that $M_{i+j}$ is minimal in $M_{i}$, and $N_{i}$ is minimal in $N_{i+j}$, by putting Lemmas 2.12 .3 together. For $M_{i+j} \subset M_{i} \subset S^{2 l-1}(1), M_{i+j}$ is minimal in $M_{i}$ indeed, since $M_{i+j}$ is minimal in $S^{2 l-1}$ (1) (c.f. [Mü80]). Similarly, $N_{i}$ is also minimal in $N_{i+j}$. Now, the proof of Theorem 1.1 is complete.

After finishing the proof of Theorem 1.1, we will continue to study the normal exponential map of isoparametric hypersurfaces constructed above to prepare for the next section.

We first consider the isoparametric function $f_{i}$ on $M_{i}$. In this case, $M_{i+1}$ is the minimal isoparametric hypersurface in $M_{i}$. At any $x \in M_{i+1}$, the unit normal $\xi(x)=P_{i+1} x$. Define a map $\phi_{t}: \mathcal{U}_{0}=M_{i+1} \rightarrow M_{i}$ by $\phi_{t}(x)=\cos t x+\sin t \xi$. In fact, it is not difficult to check that $\phi_{t}(x) \in M_{i}$, i.e., $\left\langle P_{\alpha} \phi_{t}(x), \phi_{t}(x)\right\rangle=0$ for any $0 \leq \alpha \leq i$, and hence the map $\phi_{t}$ is well-defined. Furthermore, by a direct computation, we can infer that $f_{i}\left(\phi_{t}(x)\right)=\sin 2 t$ and $\phi_{t}(x) \in \mathcal{U}_{\sin 2 t}$. For simplicity, we denote $\phi_{t}(x)$ by $x_{t}$.

For the case of the isoparametric function $g_{i}$ on $N_{i}, N_{i-1}$ is the minimal isoparametric hypersurface in $N_{i}$. At any point $x \in N_{i-1}$, the unit normal $\eta(x)=P_{i} x$. Define a map $\psi_{t}: \mathcal{V}_{0}=$ $N_{i-1} \rightarrow N_{i}$ by $\psi_{t}(x)=\cos t x+\sin t \eta$. And it is also not difficult to check that $\psi_{t}(x) \in N_{i}$, and $g_{i}\left(\psi_{t}(x)\right) \in \mathcal{V}_{\sin 2 t}$. With no possibility of confusion, we also denote $\psi_{t}(x)$ by $x_{t}$.

The following properties of maps $\phi_{t}$ and $\psi_{t}$ will be useful later.

Proposition 2.1. (1). The map $\phi_{t}$ is the normal exponential map of $M_{i+1}$ in $M_{i}$. For each $x \in M_{i+1}$, the tangent map $\left(\phi_{t}\right)_{*}: T_{x} M_{i+1} \rightarrow T_{x_{t}} M_{i}$ is given by 
a). For $X \in T_{-1}(\xi),\left(\phi_{t}\right)_{*}(X)=(\cos t+\sin t) X$;

b). For $X \in T_{1}(\xi),\left(\phi_{t}\right)_{*}(X)=(\cos t-\sin t) X$;

c). For $X \in T_{0}(\xi)$, i.e., $X=Q P_{i+1} x$, for some $Q \in \operatorname{Span}\left\{P_{0}, \cdots, P_{i}\right\},\left(\phi_{t}\right)_{*}(X)=$ $Q\left(-\sin t x+\cos t P_{i+1} x\right)$. In particular, $\left|\left(\phi_{t}\right)_{*}(X)\right|^{2}=|X|^{2}$.

(2). The map $\psi_{t}$ is the normal exponential map of $N_{i-1}$ in $N_{i}$. For each $x \in N_{i-1}$, the tangent map $\left(\psi_{t}\right)_{*}: T_{x} N_{i-1} \rightarrow T_{x_{t}} N_{i}$ is given by

a). For $X \in T_{-1}(\eta),\left(\psi_{t}\right)_{*}(X)=(\cos t+\sin t) X$;

b). For $X \in T_{1}(\eta),\left(\psi_{t}\right)_{*}(X)=(\cos t-\sin t) X$;

c). For $X \in T_{0}(\eta),\left(\psi_{t}\right)_{*}(X)=\cos t X+\sin t P_{i} X$ and $\left|\left(\psi_{t}\right)_{*}(X)\right|^{2}=|X|^{2}$.

Proof. As the proof of part (2) is similar to that of part (1), we only give the proof of part (1). Recall that the normal exponential map of $M_{i+1} \subset M_{i}$ is given by $\exp : M_{i+1} \times \mathbb{R} \rightarrow$ $M_{i}, \exp (x, t)=\exp _{x}(t \xi)$, which is the restriction of the exponential map exp of $M_{i}$ to the normal bundle of $M_{i+1}$. Observing that the curve $\phi_{t}(x)=\cos t x+\sin t \xi$ is a geodesic in $S^{2 l-1}(1)$ issuing from $x$ with initial vector $\xi(x)$ and $\phi_{t}(x) \in M_{i} \subset S^{2 l-1}(1)$, we obtain that $\exp (x, t)=\phi_{t}(x)$. Therefore, $\phi_{t}$ is exactly the normal exponential map of $M_{i+1}$ in $M_{i}$. Next, by definition of the tangent map, for each $X \in T_{x} M_{i+1},\left(\phi_{t}\right)_{*}(X)=\cos t X+\sin t P_{i+1} X$. Then part (1) of Proposition 2.1 follows immediately.

Based on Proposition 2.1, we have the following remark.

Remark 2.1. If $\sin 2 t \neq \pm 1$, the maps $\phi_{t}$ and $\psi_{t}$ are essentially diffeomorphisms from $M_{i+1}$ to the parallel hypersurface $\mathcal{U}_{\sin 2 t}$ and from $N_{i-1}$ to the parallel hypersurface $\mathcal{V}_{\sin 2 t}$, respectively. If $\sin 2 t= \pm 1$, the maps $\phi_{t}$ and $\psi_{t}$ (focal maps) are essentially submersions from $M_{i+1}$ to $\mathcal{U}_{ \pm 1}$ and from $N_{i-1}$ to $\mathcal{V}_{ \pm 1}$, respectively. However, due to Proposition 2.1 they are not Riemannian submersions.

We will conclude this section by the following result, which gives the geometry properties of the fibers of the submersions mentioned in Remark 2.1.

Proposition 2.2. (1). For $t= \pm \frac{\pi}{4}$, the maps $\phi_{ \pm \frac{\pi}{4}}: M_{i+1} \rightarrow \mathcal{U}_{ \pm 1}$ are given by $\phi_{ \pm \frac{\pi}{4}}(x)=$ $\frac{1}{\sqrt{2}}\left(x \pm P_{i+1} x\right)$ for $x \in M_{i+1}$. For any $y \in \mathcal{U}_{1}$, the fiber $F_{y}=\phi_{\frac{\pi}{4}}^{-1}(y)$ is a totally geodesic submanifold in $M_{i+1}$, and is isometric to $S^{l-i-2}\left(\frac{1}{\sqrt{2}}\right)$. For any $y^{\prime} \in \mathcal{U}_{-1}$, the fiber $F_{y^{\prime}}^{\prime}=\phi_{-\frac{\pi}{4}}^{-1}\left(y^{\prime}\right)$ is also a totally geodesic submanifold in $M_{i+1}$, and is isometric to $S^{l-i-2}\left(\frac{1}{\sqrt{2}}\right)$.

(2). For $t= \pm \frac{\pi}{4}$, the maps $\psi_{ \pm \frac{\pi}{4}}: N_{i-1} \rightarrow \mathcal{V}_{ \pm 1}$ are given by $\psi_{ \pm \frac{\pi}{4}}(x)=\frac{1}{\sqrt{2}}\left(x \pm P_{i} x\right)$ for $x \in N_{i-1}$. For any $y \in \mathcal{V}_{1}$, the fiber $F_{y}=\psi_{\frac{\pi}{4}}^{-1}(y)$ is a totally geodesic submanifold in $N_{i-1}$, and is isometric to $S^{i-1}\left(\frac{1}{\sqrt{2}}\right)$. For any $y^{\prime} \in \mathcal{V}_{-1}$, the fiber $F_{y^{\prime}}^{\prime}=\psi_{-\frac{\pi}{4}}^{-1}\left(y^{\prime}\right)$ is also a totally geodesic submanifold in $N_{i-1}$, and is isometric to $S^{i-1}\left(\frac{1}{\sqrt{2}}\right)$. 
Proof. (1). Given $y \in \mathcal{U}_{1}$, it is straightforward to verify

$$
F_{y}=\phi_{\frac{\pi}{4}}^{-1}(y)=\left\{\frac{1}{\sqrt{2}}(y+z) \mid z \in S E_{-}\left(P_{i+1}\right),\left\langle z, P_{0} y\right\rangle=\cdots=\left\langle z, P_{i} y\right\rangle=0\right\} .
$$

Consequently, it is not difficult to see that $F_{y}$ is isometric to $S^{l-i-2}\left(\frac{1}{\sqrt{2}}\right)$. Hence, the left task for us is to show that $F_{y}$ is totally geodesic in $M_{i+1}$. Denote the connections of $M_{i+1}, S^{2 l-1}(1)$ and $\mathbb{R}^{2 l}$ respectively by $\nabla, \bar{\nabla}$ and $\tilde{\nabla}$. For each $x \in F_{y}, x=\frac{1}{\sqrt{2}}(y+z)$ for some $z \in S E_{-}\left(P_{i+1}\right)$ with $\left\langle z, P_{0} y\right\rangle=\cdots=\left\langle z, P_{i} y\right\rangle=0$. Since $P_{i+1} y=y$, it is clear that $P_{0} y, \cdots, P_{i} y \in E_{-}\left(P_{i+1}\right)$. Choose $v \in T_{x}\left(F_{y}\right)$, then $v \in E_{-}\left(P_{i+1}\right),\langle v, z\rangle=0$ and $\left\langle v, P_{0} y\right\rangle=\cdots=\left\langle v, P_{i} y\right\rangle=0$. Define $c(t)=\frac{1}{\sqrt{2}}(y+\cos t z+\sin t v)$. Then $c(t)$ is a geodesic in $F_{y}$ with $c(0)=x$ and $c^{\prime}(0)=\frac{1}{\sqrt{2}} v$. By a direct computation,

$$
\begin{aligned}
\bar{\nabla}_{c^{\prime}(t)} c^{\prime}(t) & =\tilde{\nabla}_{c^{\prime}(t)} c^{\prime}(t)-\left\langle\tilde{\nabla}_{c^{\prime}(t)} c^{\prime}(t), c(t)\right\rangle c(t) \\
& =-\frac{1}{\sqrt{2}}(\cos t z+\sin t v)-\left\langle\tilde{\nabla}_{c^{\prime}(t)} c^{\prime}(t), c(t)\right\rangle c(t) \\
& =\frac{1}{2 \sqrt{2}}(y-\cos t z-\sin t v) .
\end{aligned}
$$

Since $\frac{1}{\sqrt{2}}(y-\cos t z-\sin t v)=P_{i+1} c(t)$ is a normal vector to $M_{i+1}$, we have $\nabla_{c^{\prime}(t)} c^{\prime}(t)=0$. And it means that $c(t)$ is a geodesic in $M_{i+1}$. Therefore, it follows that $F_{y}$ is totally geodesic in $M_{i+1}$.

Similarly, for $y^{\prime} \in \mathcal{U}_{-1}$, it follows

$$
F_{y^{\prime}}^{\prime}=\phi_{-\frac{\pi}{4}}^{-1}\left(y^{\prime}\right)=\left\{\frac{1}{\sqrt{2}}\left(y^{\prime}+z\right) \mid z \in S E_{+}\left(P_{i+1}\right),\left\langle z, P_{0} y^{\prime}\right\rangle=\cdots=\left\langle z, P_{i} y^{\prime}\right\rangle=0\right\},
$$

and $F_{y^{\prime}}^{\prime}$ is totally geodesic in $M_{i+1}$, but the detailed proof is omitted here.

(2). For $y \in \mathcal{V}_{1}$, the fiber is given by

$$
F_{y}=\psi_{\frac{\pi}{4}}^{-1}(y)=\left\{\frac{1}{\sqrt{2}}(y+z) \mid z \in \operatorname{Span}\left\{P_{0} y, \cdots, P_{i-1} y\right\}\right\},
$$

and thus $F_{y}$ is isometric to $S^{i-1}\left(\frac{1}{\sqrt{2}}\right)$. Next, we will show $F_{y}$ is totally geodesic in $N_{i-1}$. Choose any $x \in F_{y}$ and $v \in T_{x} F_{y}$, then $x=\frac{1}{\sqrt{2}}(y+z)$ for some $z \in \operatorname{Span}\left\{P_{0} y, \cdots, P_{i-1} y\right\}$, and $v \in$ $\operatorname{Span}\left\{P_{0} y, \cdots, P_{i-1} y\right\}$ with $\langle v, z\rangle=0$. Now, we can define $c(t)=\frac{1}{\sqrt{2}}(y+\cos t z+\sin t v)$. Clearly, $c(t)$ is a geodesic in $F_{y}$ with $c(0)=x$ and $c^{\prime}(0)=\frac{1}{\sqrt{2}} v$. It follows that

$$
\begin{aligned}
\bar{\nabla}_{c^{\prime}(t)} c^{\prime}(t) & =\tilde{\nabla}_{c^{\prime}(t)} c^{\prime}(t)-\left\langle\tilde{\nabla}_{c^{\prime}(t)} c^{\prime}(t), c(t)\right\rangle c(t) \\
& =-\frac{1}{\sqrt{2}}(\cos t z+\sin t v)-\left\langle\tilde{\nabla}_{c^{\prime}(t)} c^{\prime}(t), c(t)\right\rangle c(t) \\
& =\frac{1}{2 \sqrt{2}}(y-\cos t z-\sin t v),
\end{aligned}
$$

where the connections of $N_{i-1}, S^{2 l-1}(1)$ and $\mathbb{R}^{2 l}$ are denoted by $\nabla, \bar{\nabla}$ and $\tilde{\nabla}$, respectively. And then $\nabla_{c^{\prime}(t)} c^{\prime}(t)=0$, because $\frac{1}{\sqrt{2}}(y-\cos t z-\sin t v)=P_{i} c(t)$ is a normal vector to $N_{i-1}$. That is to say, $c(t)$ is a geodesic in $N_{i-1}$. Hence, $F_{y}$ is totally geodesic in $N_{i-1}$. 
For the case $t=-\frac{\pi}{4}$, the proof is analogous to the above and we will not go into the details. In fact, for $y^{\prime} \in \mathcal{V}_{-1}$, the fiber is given by

$$
F_{y^{\prime}}^{\prime}=\psi_{-\frac{\pi}{4}}^{-1}\left(y^{\prime}\right)=\left\{\frac{1}{\sqrt{2}}\left(y^{\prime}+z\right) \mid z \in \operatorname{Span}\left\{P_{0} y^{\prime}, \cdots, P_{i-1} y^{\prime}\right\}\right\}
$$

and $F_{y^{\prime}}^{\prime}$ is totally geodesic in $N_{i-1}$.

Remark 2.2. Corollary 1.1 follows from Theorem 1.1 and Proposition 2.2.

\section{Eigenvalue estimates}

Based on the isoparametric foliations constructed in Theorem 1.1 we intend to prove Theorem 1.2 on eigenvalues estimate of the Laplacian in this section. We first recall a crucial theorem which has been used in [Mu88], [TY13] and [TXY14].

Theorem (Chavel and Feldman [CF78], Ozawa [Oz81]) Let $V$ be a closed, connected smooth Riemannian manifold and $W$ a closed submanifold of $V$. For any sufficiently small $\varepsilon>0$, set $W(\varepsilon)=\{x \in V: \operatorname{dist}(x, W)<\varepsilon\}$. Let $\lambda_{k}^{D}(\varepsilon)(k=1,2, \cdots)$ be the $k$-th eigenvalue of the Laplace-Beltrami operator on $V-W(\varepsilon)$ under the Dirichlet boundary condition. If $\operatorname{dim} V \geq \operatorname{dim} W+2$, then for any $k=0,1, \cdots$

$$
\lim _{\varepsilon \rightarrow 0} \lambda_{k+1}^{D}(\varepsilon)=\lambda_{k}(V)
$$

It is necessary to point out that the proof of part (2) of Theorem 1.2 is analogous to that of part (1), so the detailed proof will be only given for part (1).

\section{Proof of Theorem 1.2 (1):}

Proof. a). Consider the isoparametric foliation on $M_{i}$ given by the function $f_{i}$, provided that $0 \leq i \leq m-1$ and $l-i-3>0$. For sufficiently small $\varepsilon>0$, set

$$
\mathcal{U}(\varepsilon)=\bigcup_{t \in\left[-\frac{\pi}{4}+\varepsilon, \frac{\pi}{4}-\varepsilon\right]} \mathcal{U}_{\sin 2 t}
$$

Actually, $\mathcal{U}(\varepsilon)$ is a domain of $M_{i}$ obtained by excluding $\varepsilon$-neighborhoods of $\mathcal{U}_{1}$ and $\mathcal{U}_{-1}$. Thus, by the theorem of Chavel-Feldman and Ozawa,

$$
\lim _{\varepsilon \rightarrow 0} \lambda_{k+1}^{D}(\mathcal{U}(\varepsilon))=\lambda_{k}\left(M_{i}\right)
$$

Next we will estimate $\lambda_{k+1}^{D}(\mathcal{U}(\varepsilon))$ from above in terms of $\lambda_{k}\left(M_{i+1}\right)$ by making use of the minimax principle.

According to part (1) of Proposition 2.1, the volume element of $\mathcal{U}(\varepsilon)$ can be expressed by the volume element of $M_{i+1}$ as

$$
d \mathcal{U}(\varepsilon)=(\cos t+\sin t)^{l-i-2}(\cos t-\sin t)^{l-i-2} d t d M_{i+1}=(\cos 2 t)^{l-i-2} d t d M_{i+1} .
$$


Let $h$ be a nonnegative, increasing smooth function on $[0, \infty)$ satisfying $h=1$ on $[2, \infty)$ and $h=0$ on $[0,1]$. For sufficiently small $\varrho>0$, define a nonnegative smooth function $\Psi_{\varrho}$ on $\left[-\frac{\pi}{2}, \frac{\pi}{2}\right]$ by

(i) $\Psi_{\varrho}(x)=1$ on $\left[-\frac{\pi}{2}+2 \varrho, \frac{\pi}{2}-2 \varrho\right]$,

(ii) $\Psi_{\varrho}(x)=h\left(\frac{\frac{\pi}{2}-x}{\varrho}\right)$ on $\left[\frac{\pi}{2}-2 \varrho, \frac{\pi}{2}\right]$,

(iii) $\Psi_{\varrho}$ is symmetric with respect to $x=0$.

Then $\left|\Psi_{\varrho}^{\prime}(x)\right| \leq \frac{1}{\varrho} C$ for $x \in\left[-\frac{\pi}{2}, \frac{\pi}{2}\right]$, where $C=\sup \left\{h^{\prime}(x) \mid x \in[0, \infty)\right\}$.

Let $\varphi_{k}$ be the $k$-th eigenfunctions on $M_{i+1}$ which are orthogonal to each other with respect to the square integral inner product on $M_{i+1}$ and $L_{k+1}=\operatorname{Span}\left\{\varphi_{0}, \varphi_{1}, \cdots, \varphi_{k}\right\}$. For each fixed $t \in\left[-\frac{\pi}{4}+\varepsilon, \frac{\pi}{4}-\varepsilon\right]$, denote $\pi=\pi_{t}=\phi_{t}^{-1}: \mathcal{U}_{\sin 2 t} \rightarrow M_{i+1}$. Given any $\varphi \in L_{k+1}$, we can define a function $\Phi_{\varepsilon}$ on $\mathcal{U}(\varepsilon)$ by $\Phi_{\varepsilon}(x)=\Psi_{2 \varepsilon}(2 t)(\varphi \circ \pi)(x)$, where $t$ is determined by $x \in \mathcal{U}_{\sin 2 t}$ and $t \in\left[-\frac{\pi}{4}+\varepsilon, \frac{\pi}{4}-\varepsilon\right]$. It is clear that $\Phi_{\varepsilon}$ is a smooth function on $\mathcal{U}(\varepsilon)$ satisfying the Dirichlet boundary condition.

By the mini-max principle, we can infer that

$$
\lambda_{k+1}^{D}(\mathcal{U}(\varepsilon)) \leq \sup _{\varphi \in L_{k+1}} \frac{\left\|\nabla \Phi_{\varepsilon}\right\|_{2}^{2}}{\left\|\Phi_{\varepsilon}\right\|_{2}^{2}} .
$$

Now, we will estimate the term $\frac{\left\|\nabla \Phi_{\varepsilon}\right\|_{2}^{2}}{\left\|\Phi_{\varepsilon}\right\|_{2}^{2}}$. Since the normal geodesic starting from $M_{i+1}$ is perpendicular to any parallel hypersurface $\mathcal{U}_{c}$, it follows that

$$
\left\|\nabla \Phi_{\varepsilon}\right\|_{2}^{2}=\int_{\mathcal{U}(\varepsilon)} 4\left(\Psi_{2 \varepsilon}^{\prime}(2 t)\right)^{2}(\varphi \circ \pi)^{2} d \mathcal{U}(\varepsilon)+\int_{\mathcal{U}(\varepsilon)}\left(\Psi_{2 \varepsilon}(2 t)\right)^{2}|\nabla(\varphi \circ \pi)|^{2} d \mathcal{U}(\varepsilon)
$$

Moreover,

$$
\begin{aligned}
\left\|\Phi_{\varepsilon}\right\|_{2}^{2} & =\int_{\mathcal{U}(\varepsilon)} \Psi_{2 \varepsilon}^{2}(2 t)(\varphi \circ \pi)^{2} d \mathcal{U}(\varepsilon) \\
& =\int_{-\frac{\pi}{4}+\varepsilon}^{\frac{\pi}{4}+\epsilon} \int_{M_{i+1}} \Psi_{2 \varepsilon}^{2}(2 t)(\cos 2 t)^{l-i-2} \varphi^{2} d t d M_{i+1} \\
& =\frac{\|\varphi\|_{2}^{2}}{2} \int_{-\frac{\pi}{2}+2 \varepsilon}^{\frac{\pi}{2}-2 \varepsilon} \Psi_{2 \varepsilon}^{2}(\tau)(\cos \tau)^{l-i-2} d \tau,
\end{aligned}
$$

and thus,

where

$$
\frac{\left\|\nabla \Phi_{\varepsilon}\right\|_{2}^{2}}{\left\|\Phi_{\varepsilon}\right\|_{2}^{2}}=I(\varepsilon)+I I(\varepsilon),
$$

$$
\begin{aligned}
I(\varepsilon) & =\frac{\int_{\mathcal{U}(\varepsilon)} 4\left(\Psi_{2 \varepsilon}^{\prime}(2 t)\right)^{2}(\varphi \circ \pi)^{2} d \mathcal{U}(\varepsilon)}{\int_{\mathcal{U}(\varepsilon)} \Psi_{2 \varepsilon}^{2}(2 t)(\varphi \circ \pi)^{2} d \mathcal{U}(\varepsilon)} \\
& =\frac{4 \int_{-\frac{\pi}{2}+2 \varepsilon}^{\frac{\pi}{2}-2 \varepsilon}\left(\Psi_{2 \varepsilon}^{\prime}(\tau)\right)^{2}(\cos \tau)^{l-i-2} d \tau}{\int_{-\frac{\pi}{2}+2 \varepsilon}^{\frac{\pi}{2}-2 \varepsilon} \Psi_{2 \varepsilon}^{2}(\tau)(\cos \tau)^{l-i-2} d \tau},
\end{aligned}
$$


and

$$
I I(\varepsilon)=\frac{\int_{\mathcal{U}(\varepsilon)}\left(\Psi_{2 \varepsilon}(2 t)\right)^{2}|\nabla(\varphi \circ \pi)|^{2} d \mathcal{U}(\varepsilon)}{\int_{\mathcal{U}(\varepsilon)} \Psi_{2 \varepsilon}^{2}(2 t)(\varphi \circ \pi)^{2} d \mathcal{U}(\varepsilon)}
$$

Observing that if $l-i-3>0$,

$$
\begin{aligned}
& \int_{-\frac{\pi}{2}+2 \varepsilon}^{\frac{\pi}{2}-2 \varepsilon}\left(\Psi_{2 \varepsilon}^{\prime}(\tau)\right)^{2}(\cos \tau)^{l-i-2} d \tau \\
\leq & \int_{-\frac{\pi}{2}+2 \varepsilon}^{-\frac{\pi}{2}+4 \varepsilon}\left(\Psi_{2 \varepsilon}^{\prime}(\tau)\right)^{2}(\cos \tau)^{l-i-2} d \tau+\int_{\frac{\pi}{2}-4 \varepsilon}^{\frac{\pi}{2}-2 \varepsilon}\left(\Psi_{2 \varepsilon}^{\prime}(\tau)\right)^{2}(\cos \tau)^{l-i-2} d \tau \\
\leq & \int_{-\frac{\pi}{2}+2 \varepsilon}^{-\frac{\pi}{2}+4 \varepsilon} \frac{C^{2}}{4 \varepsilon^{2}} \cos ^{2} \tau d \tau+\int_{\frac{\pi}{2}-4 \varepsilon}^{\frac{\pi}{2}-2 \varepsilon} \frac{C^{2}}{4 \varepsilon^{2}} \cos ^{2} \tau d \tau,
\end{aligned}
$$

we deduce $\lim _{\varepsilon \rightarrow 0} I(\varepsilon)=0$.

It remains to consider the term $I I(\varepsilon)$. Decompose

$$
\nabla \varphi=Z_{1}+Z_{2}+Z_{3} \in T_{-1}(\xi) \oplus T_{1}(\xi) \oplus T_{0}(\xi)=T M_{i+1} .
$$

By definition, $\langle\nabla(\varphi \circ \pi), X\rangle=\left\langle\nabla \varphi, \pi_{*} X\right\rangle$ for $X \in T \mathcal{U}_{\sin 2 t}$. From Proposition 2.1,

$$
|\nabla(\varphi \circ \pi)|^{2}=\frac{1}{\kappa_{1}^{2}}\left|Z_{1}\right|^{2}+\frac{1}{\kappa_{2}^{2}}\left|Z_{2}\right|^{2}+\frac{1}{\kappa_{3}^{2}}\left|Z_{3}\right|^{2}
$$

where $\kappa_{1}=\cos t+\sin t, \kappa_{1}=\cos t-\sin t$, and $\kappa_{3}=1$. Define

$$
K_{1}=\int_{-\frac{\pi}{4}}^{\frac{\pi}{4}} \frac{(\cos 2 t)^{l-i-2}}{\kappa_{1}^{2}} d t, K_{2}=\int_{-\frac{\pi}{4}}^{\frac{\pi}{4}} \frac{(\cos 2 t)^{l-i-2}}{\kappa_{2}^{2}} d t, K_{3}=G=\int_{-\frac{\pi}{4}}^{\frac{\pi}{4}}(\cos 2 t)^{l-i-2} d t,
$$

and $K=\max \left\{K_{1}, K_{2}, K_{3}\right\}$. Then

$$
\lim _{\varepsilon \rightarrow 0} I I(\varepsilon)=\frac{\sum_{\alpha=1}^{3} K_{\alpha}\left\|Z_{\alpha}\right\|_{2}^{2}}{\|\varphi\|_{2}^{2} G} \leq \frac{K}{G} \frac{\|\nabla \varphi\|_{2}^{2}}{\|\varphi\|_{2}^{2}} .
$$

Furthermore,

$$
\lambda_{k}\left(M_{i}\right)=\lim _{\varepsilon \rightarrow 0} \lambda_{k+1}^{D}(\mathcal{U}(\varepsilon)) \leq \lim _{\varepsilon \rightarrow 0} \sup _{\varphi \in L_{k+1}} \frac{\left\|\nabla \Phi_{\varepsilon}\right\|_{2}^{2}}{\left\|\Phi_{\varepsilon}\right\|_{2}^{2}} \leq \frac{K}{G} \lambda_{k}\left(M_{i+1}\right) .
$$

A direct computation yields

and the inequality a) of Theorem 1.2 (1) follows.

$$
\frac{K}{G}=\frac{K_{1}}{G}=\frac{l-i-2}{l-i-3}
$$

b). According to Proposition 2.1 and 2.2 the map $\phi_{\frac{\pi}{4}}: M_{i+1} \rightarrow \mathcal{U}_{1}$ is a smooth submersion (but not a Riemannian submersion), and for any $y \in \mathcal{U}_{1}$, the fiber $F_{y}=\phi_{\frac{\pi}{4}}^{-1}(y)$, isometric to $S^{l-i-2}\left(\frac{1}{\sqrt{2}}\right)$, is a totally geodesic submanifold in $M_{i+1}$. Moreover, For each $y \in \mathcal{U}_{1}$, at a point $x \in \phi_{\frac{\pi}{4}}^{-1}(y)$, we have a decomposition $T_{x} M_{i+1}=T_{-1}(\xi) \oplus T_{1}(\xi) \oplus T_{0}(\xi)$, and

$$
\begin{aligned}
& \left(\phi_{\frac{\pi}{4}}\right)_{*}(X)=\left(\cos \frac{\pi}{4}+\sin \frac{\pi}{4}\right) X=\sqrt{2} X, \text { for } X \in T_{-1}(\xi) ; \\
& \left(\phi_{\frac{\pi}{4}}\right)_{*}(X)=\left(\cos \frac{\pi}{4}-\sin \frac{\pi}{4}\right) X=0, \text { for } X \in T_{1}(\xi) ;
\end{aligned}
$$


$\left(\phi_{\frac{\pi}{4}}\right)_{*}(X)=Q\left(-\sin \frac{\pi}{4} x+\cos \frac{\pi}{4} P_{i+1} x\right)$, for $X \in T_{0}(\xi)$, i.e., $X=Q P_{i+1} x$ with $Q \in \operatorname{Span}\left\{P_{0}, \cdots, P_{i}\right\}$. In particular, $\left|\left(\phi_{\frac{\pi}{4}}\right)_{*}(X)\right|^{2}=|X|^{2}$.

Using these facts, we will show the inequality b) in Theorem 1.2 (1) as follows. Let $\varphi_{k}$ be the $k$-th eigenfunctions on $\mathcal{U}_{1}$ which are orthogonal to each other with respect to the square integral inner product on $\mathcal{U}_{1}$ and $L_{k+1}=\operatorname{Span}\left\{\varphi_{0}, \varphi_{1}, \cdots, \varphi_{k}\right\}$. For any $\varphi \in L_{k+1}$, define a function $\Phi$ on $M_{i+1}$ by $\Phi(x)=\left(\varphi \circ \phi_{\frac{\pi}{4}}\right)(x)$. By the min-max principle again, we get

$$
\lambda_{k}\left(M_{i+1}\right) \leq \sup _{\varphi \in L_{k+1}} \frac{\|\nabla \Phi\|_{2}^{2}}{\|\Phi\|_{2}^{2}}=\sup _{\varphi \in L_{k+1}} \frac{\int_{M_{i+1}}|\nabla \Phi|^{2} d M_{i+1}}{\int_{M_{i+1}} \Phi^{2} d M_{i+1}} .
$$

Hence, the term $|\nabla \Phi|^{2}$ has to be estimated. In fact, by the properties of $\left(\phi_{\frac{\pi}{4}}\right)_{*}$ described above, it follows that $|\nabla \Phi|_{x}^{2} \leq 2|\nabla \varphi|_{y}^{2}$. Then

$$
\begin{aligned}
\int_{M_{i+1}}|\nabla \Phi|^{2} d M_{i+1} & =\int_{M_{i+1}} \frac{|\nabla \Phi|^{2}}{(\sqrt{2})^{l-i-2}}\left(\phi_{\frac{\pi}{4}}\right)^{*}\left(d \mathcal{U}_{1}\right) d S^{l-i-2}\left(\frac{1}{\sqrt{2}}\right) \\
& \leq \int_{M_{i+1}} \frac{2|\nabla \varphi|^{2}}{(\sqrt{2})^{l-i-2}}\left(\phi_{\frac{\pi}{4}}\right)^{*}\left(d \mathcal{U}_{1}\right) d S^{l-i-2}\left(\frac{1}{\sqrt{2}}\right) \\
& =\frac{2}{(\sqrt{2})^{l-i-2}} \operatorname{Vol}\left(S^{l-i-2}\left(\frac{1}{\sqrt{2}}\right)\right) \int_{\mathcal{U}_{1}}|\nabla \varphi|^{2} d \mathcal{U}_{1},
\end{aligned}
$$

and

$$
\begin{aligned}
\int_{M_{i+1}} \Phi^{2} d M_{i+1} & =\int_{M_{i+1}} \frac{\Phi^{2}}{(\sqrt{2})^{l-i-2}}\left(\phi_{\frac{\pi}{4}}\right)^{*}\left(d \mathcal{U}_{1}\right) d S^{l-i-2}\left(\frac{1}{\sqrt{2}}\right) \\
& =\int_{M_{i+1}} \frac{\varphi^{2}}{(\sqrt{2})^{l-i-2}}\left(\phi_{\frac{\pi}{4}}\right)^{*}\left(d \mathcal{U}_{1}\right) d S^{l-i-2}\left(\frac{1}{\sqrt{2}}\right) \\
& =\frac{1}{(\sqrt{2})^{l-i-2}} \operatorname{Vol}\left(S^{l-i-2}\left(\frac{1}{\sqrt{2}}\right)\right) \int_{\mathcal{U}_{1}} \varphi^{2} d \mathcal{U}_{1}
\end{aligned}
$$

Therefore,

$$
\lambda_{k}\left(M_{i+1}\right) \leq \sup _{\varphi \in L_{k+1}} \frac{\|\nabla \Phi\|_{2}^{2}}{\|\Phi\|_{2}^{2}} \leq 2 \sup _{\varphi \in L_{k+1}} \frac{\|\nabla \varphi\|_{2}^{2}}{\|\varphi\|_{2}^{2}}=2 \lambda_{k}\left(\mathcal{U}_{1}\right)=2 \lambda_{k}\left(S^{l-1}(1)\right)
$$

as required.

Remark 3.1. (1). According to [TY13], $\lambda_{k}\left(M_{i}\right) \geq \frac{l-i-2}{l-1} \lambda_{k}\left(S^{2 l-1}(1)\right)$, for $i \geq 2$. Combining the inequality a) of Theorem 1.2 (1) with the inequality of [TY13] for the $i$ case, we can infer that $\lambda_{k}\left(M_{i+1}\right) \geq \frac{l-i-3}{l-1} \lambda_{k}\left(S^{2 l-1}(1)\right)$, which is the inequality of [TY13] for the $i+1$ case.

(2). For the isoparametric foliation on $M_{i}$ determined by $f_{i}$, as in Section 3 of [TY13], we can also obtain the inequality $\lambda_{k}\left(M_{i}\right) \leq \frac{2(l-i-2)}{l-i-3} \lambda_{k}\left(S^{l-1}(1)\right)$, which is a consequence of the inequalities a) and b) of Theorem 1.2(1).

(3). Using the method of the proof for the inequality $b$ ) of Theorem 1.2 (1), for a minimal isoparametric hypersurface $M$ in the unit sphere with $g=4$, multiplicities $\left(m_{1}, m_{2}\right)$ and focal 
submanifolds $M_{+}$and $M_{-}$of codimension $m_{1}+1$ and $m_{2}+1$ respectively, we can show that $\lambda_{k}(M) \leq \frac{m_{1}+m_{2}}{m_{2}} \lambda_{k}\left(M_{+}\right)$and $\lambda_{k}(M) \leq \frac{m_{1}+m_{2}}{m_{1}} \lambda_{k}\left(M_{-}\right)$.

(4). The remarks above on the isoparametric function $f_{i}$ on $M_{i}$ are also available for the case of the isoparametric function $g_{i}$ on $N_{i}$.

Next, let us focus on eigenvalue estimates in a specific case. As is well known, for $g=4$, $\left(m_{1}, m_{2}\right)=(4,3)$, there are exactly two non-congruent families (one is homogenous and the other is not) of isoparametric hypersurfaces of OT-FKM type. For the homogeneous case, Tang, Xie and Yan [TXY14] determined the first eigenvalue of the focal submanifold $M_{+}^{10}$, that is, $\lambda_{1}\left(M_{+}^{10}\right)=10$. However, for the inhomogeneous case, the corresponding work is still open. To study the spectrum of the focal submanifold in this case, we establish the following result.

Proposition 3.1. Let $\left\{P_{0}, \cdots, P_{4}\right\}$ on $\mathbb{R}^{16}$ be a symmetric Clifford system.

1). For the case $P_{0} \cdots P_{4}= \pm I_{16}$, the corresponding isoparametric foliation is homogenous and the focal submanifold $M_{+}^{10}$ is isometric to $\mathrm{Sp}(2)$ with certain bi-invariant metric. In particular, $\lambda_{17}\left(M_{+}^{10}\right)=16$.

2). For the case $P_{0} \cdots P_{4} \neq \pm I_{16}$, the corresponding isoparametric foliation is inhomogeneous and the focal submanifold $\tilde{M}_{+}^{10}$ is only diffeomorphic to $S^{3} \times S^{7}$, but not isometric to the product of two round spheres. Moreover, $\lambda_{17}\left(\tilde{M}_{+}^{10}\right) \leq 12$.

Proof. 1). Up to orthogonal transformations, the symmetric Clifford system in this case can be chosen as follows. First, using the multiplication of quaternions, we can define three orthogonal transformations $E_{1}, E_{2}, E_{3}$ on $\mathbb{R}^{8}=\mathbb{H} \oplus \mathbb{H}$, where any point in $\mathbb{R}^{8}$ is considered as two quaternions. For $u=\left(u_{1}, u_{2}\right) \in \mathbb{H}^{2}$,

$$
\begin{aligned}
& E_{1}(u)=\left(\mathrm{i} u_{1}, \mathrm{i} u_{2}\right), \\
& E_{2}(u)=\left(\mathrm{j} u_{1}, \mathrm{j} u_{2}\right), \\
& E_{3}(u)=\left(\mathrm{k} u_{1}, \mathrm{k} u_{2}\right) .
\end{aligned}
$$

Furthermore, by identifying $\mathbb{R}^{16}$ with $\mathbb{H}^{4}$, we can define

$$
\begin{aligned}
& P_{0}(u, v)=(u,-v), \\
& P_{1}(u, v)=(v, u), \\
& P_{2}(u, v)=\left(E_{1} v,-E_{1} u\right), \\
& P_{3}(u, v)=\left(E_{2} v,-E_{2} u\right), \\
& P_{4}(u, v)=\left(E_{3} v,-E_{3} u\right),
\end{aligned}
$$

for $(u, v)=\left(u_{1}, u_{2} ; v_{1}, v_{2}\right) \in \mathbb{H}^{4}=\mathbb{R}^{16}$. Clearly, $P_{0} P_{1} \cdots P_{4}=$ Id. According to [FKM81], the corresponding isoparametric foliation is homogeneous, with one of the focal submanifolds $M_{+}^{10}=\left\{x \in S^{15}(1) \mid\left\langle P_{\alpha} x, x\right\rangle=0,0 \leq \alpha \leq 4\right\}$. It is not difficult to show $x=(u, v)=$ 
$\left(u_{1}, u_{2} ; v_{1}, v_{2}\right) \in M_{+}^{10}$ if and only if

$$
|u|=|v|=\frac{1}{\sqrt{2}}, \quad u_{1} \overline{v_{1}}+u_{2} \overline{v_{2}}=0
$$

Thus we can define a map $G: M_{+}^{10} \rightarrow \operatorname{Sp}(2)$ by $G\left(u_{1}, u_{2} ; v_{1}, v_{2}\right)=\sqrt{2}\left(\begin{array}{cc}u_{1} & u_{2} \\ v_{1} & v_{2}\end{array}\right)$. It is evident that $G$ is a diffeomorphism. Furthermore, it is an isometry if $\mathrm{Sp}(2)$ is equipped with the biinvariant metric normalized such that the tangent vector $\left(\begin{array}{cc}\sqrt{2} \mathrm{i} & 0 \\ 0 & 0\end{array}\right) \in T_{I} \operatorname{Sp}(2)$ has unit length. For this metric, the spectrum of $\mathrm{Sp}(2)$ can be determined completely (c.f. [BM77] and ["Fe80]). Particularly, $\lambda_{17}\left(M_{+}^{10}\right)=16$.

2). Also using the multiplication of quaternions, we can define another three orthogonal transformations $E_{1}, E_{2}, E_{3}$ on $\mathbb{R}^{8}=\mathbb{H} \oplus \mathbb{H}$. For $u=\left(u_{1}, u_{2}\right) \in \mathbb{H}^{2}$,

$$
\begin{aligned}
& E_{1}(u)=\left(\mathrm{i} u_{1},-\mathrm{i} u_{2}\right), \\
& E_{2}(u)=\left(\mathrm{j} u_{1},-\mathrm{j} u_{2}\right), \\
& E_{3}(u)=\left(\mathrm{k} u_{1},-\mathrm{k} u_{2}\right) .
\end{aligned}
$$

And similarly define

$$
\begin{aligned}
& P_{0}(u, v)=(u,-v), \\
& P_{1}(u, v)=(v, u), \\
& P_{2}(u, v)=\left(E_{1} v,-E_{1} u\right), \\
& P_{3}(u, v)=\left(E_{2} v,-E_{2} u\right), \\
& P_{4}(u, v)=\left(E_{3} v,-E_{3} u\right),
\end{aligned}
$$

for $(u, v)=\left(u_{1}, u_{2} ; v_{1}, v_{2}\right) \in \mathbb{H}^{4}=\mathbb{R}^{16}$. In this case, $P_{0} P_{1} \cdots P_{4} \neq \pm \mathrm{Id}$. According to [FKM81], the corresponding isoparametric foliation is inhomogeneous. Now, $x=(u, v)=$ $\left(u_{1}, u_{2} ; v_{1}, v_{2}\right) \in \tilde{M}_{+}^{10}$ (one of the focal submanifolds) if and only if

$$
|u|=|v|=\frac{1}{\sqrt{2}}, \quad v_{1} \overline{u_{1}}+u_{2} \overline{v_{2}}=0 .
$$

Moreover, an explicit diffeomorphism $F$ from $\tilde{M}_{+}^{10}$ to $S^{7}\left(\frac{1}{\sqrt{2}}\right) \times S^{3}(1)$ can be constructed by

$$
F\left(u_{1}, u_{2} ; v_{1}, v_{2}\right)=\left(u_{1}, u_{2} ; 2\left(\overline{u_{2}} v_{1}-\overline{v_{2}} u_{1}\right)\right) .
$$

Observe that $F$ is not an isometry from $\tilde{M}_{+}^{10}$ to $S^{7}\left(\frac{1}{\sqrt{2}}\right) \times S^{3}(1)$ with the standard product metric. In light of the diffeomorphism $F$, it is not difficult to show the four coordinate components of $S^{3}(1)$, the second factor of $S^{7}\left(\frac{1}{\sqrt{2}}\right) \times S^{3}(1)$, provide 4 eigenfunctions on $\tilde{M}_{+}^{10}$ with the same eigenvalue 12. For instance, given $\Phi: \mathbb{R}^{16} \rightarrow \mathbb{R}$ by $\Phi\left(u_{1}, u_{2} ; v_{1}, v_{2}\right)=\left\langle u_{1}, v_{2}\right\rangle-\left\langle u_{2}, v_{1}\right\rangle$, and $\varphi: \tilde{M}_{+}^{10} \rightarrow \mathbb{R}$ by $\varphi:=\left.\Phi\right|_{\tilde{M}_{+}^{10}}$, then $\Delta \varphi=-12 \varphi$ by a direct computation. On the other hand, since $\tilde{M}_{+}^{10}$ is minimal in $S^{15}(1)$, the $\mathbb{R}^{16}$-components give 16 eigenfunctions with the same eigenvalue 10 . These arguments imply that $\lambda_{17}\left(\tilde{M}_{+}^{10}\right) \leq 12$ as required. 
Remark 3.2. From the view point of representation theory, it is worth mentioning that the symmetric Clifford system $\left\{P_{0}, \cdots, P_{4}\right\}$ on $\mathbb{R}^{16}$ with $P_{0} \cdots P_{4}= \pm I_{16}$ cannot be extended. However, for the symmetric Clifford system $\left\{P_{0}, \cdots, P_{4}\right\}$ on $\mathbb{R}^{16}$ with $P_{0} \cdots P_{4} \neq \pm I_{16}$, it can be extend to a symmetric Clifford system $\left\{P_{0}, \cdots, P_{4}, P_{5}\right\}$ on $\mathbb{R}^{16}$ indeed.

\section{ISOPARAMETRIC FOLIATION AND HARMONIC MAP}

This section will be concerned with harmonic maps and their energy-stability via isoparametric focal maps. We will prove Theorem 1.4 and Proposition 1.1 on harmonic maps, and then investigate the stability of these harmonic maps. For convenience, we begin with recalling the following basic fact.

Lemma 4.1. [EL78] For Riemannian manifolds $M, N$ and $P$, let $f$ be a smooth map from $M$ to $N$, and $i$ an isometric immersion from $N$ into $P$. Define $F=i \circ f: M \rightarrow P$. Then $f$ is harmonic if and only if the tension field of $F$ is normal to $N$.

We are now ready to prove Theorem 1.4

\section{Proof of Theorem 1.4:}

Proof. We only consider part (1) of this theorem. Let $\left\{P_{0}, \cdots, P_{m}\right\}$ be a symmetric Clifford system on $\mathbb{R}^{2 l}$. For $0 \leq i \leq m-1$, the focal maps $\phi_{ \pm \frac{\pi}{4}}: M_{i+1} \rightarrow \mathcal{U}_{ \pm 1}=S E_{ \pm}\left(P_{i+1}\right)$ given by

$$
\phi_{ \pm \frac{\pi}{4}}(x)=\frac{1}{\sqrt{2}}\left(x \pm P_{i+1} x\right), x \in M_{i+1}
$$

are smooth submersions, due to Proposition 2.1 Since $M_{i+1}$ is minimal in $S^{2 l-1}(1)$, by using Takahashi Theorem, we get $\Delta x=-(2 l-i-3) x$. It follows that

$$
\triangle \Phi_{ \pm}=-(2 l-i-3) \Phi_{ \pm},
$$

where $\Phi_{ \pm}=i_{ \pm} \circ \phi_{ \pm \frac{\pi}{4}}$ and $i_{ \pm}: \mathcal{U}_{ \pm 1}=S E_{ \pm}\left(P_{i+1}\right) \rightarrow E_{ \pm}\left(P_{i+1}\right) \cong \mathbb{R}^{l}$ are inclusion maps. Now, the proof follows from Lemma4.1

Remark 4.1. By definition, a smooth map $f$ between Riemannian manifolds $M$ and $N$ is called a harmonic morphism if the pull back of any local harmonic function on $N$ by $f$ is also a local harmonic function on $M$. It is well known that a smooth map is a harmonic morphism if and only if it is simultaneously harmonic and weakly horizontally conformal (see, for example, [EL78]). In particular, a submersive harmonic morphism should be a horizontally conformal map. According to Proposition 2.1 the eigenmaps determined by Theorem 1.4 are not harmonic morphisms.

Remark 4.2. Recall that a harmonic map $f$ is called (energy) stable if every second variation of the energy functional at $f$ is nonnegative. Due to [Le82] and [Pe84], for $n \geq 3$, any stable harmonic map from any compact Riemannian manifold $M^{m}$ to $S^{n}(1)$ is constant. Thus, for $l \geq 4$, the eigenmaps constructed in Theorem 1.4 are unstable. Moreover, for $l=3$, the 
eigenmaps we constructed are also unstable, because that any stable harmonic map from any compact Riemannian manifold $M^{m}$ to $S^{2}(1)$ is a harmonic morphism by one of the main results in [Ch96].

Next, we deal with the case of isoparametric hypersurfaces in unit spheres.

\section{Proof of Proposition 1.1;}

Proof. Given an isoparametric hypersurface $M^{n}$ in $S^{n+1}(1)$ and $M_{+}, M_{-}$the focal submanifolds with codimension $m_{1}+1, m_{2}+1$, respectively. Choose a smooth field $\xi$ of unit normals to $M$. To prove this proposition, it is sufficient to consider one focal map $\varphi: M \rightarrow M_{+}$. In this case, for each $x \in M \subset S^{n+1}(1), \varphi(x)=\cos \theta x+\sin \theta \xi(x)$ for certain $\theta$. Due to Lemma4.1, we need to compute the Laplacian of $F$ on $M$, where $F=i \circ \varphi$ and $i: M_{+} \rightarrow \mathbb{R}^{n+2}$ is the inclusion map. Using the fact that $M$ has constant mean curvature in $S^{n+1}(1)$ and the Codazzi equation, we compute directly and get

$$
\left\{\begin{array}{c}
\triangle x=-n x+H \xi, \\
\triangle \xi=H x-|B|^{2} \xi,
\end{array}\right.
$$

where $B, H$ and $|B|^{2}$ are second fundamental form, mean curvature with respect to $\xi$ and squared norm of the second fundamental form for the isoparametric hypersurface $M$ in $S^{n+1}(1)$, respectively. Thus $\Delta F=(-\cos \theta n++\sin \theta H) x+\left(\cos \theta H-\sin \theta|B|^{2}\right) \xi$, which is normal to $M_{+}$. It follows from Lemma 4.1 that $\varphi$ is harmonic as desired.

Remark 4.3. It is interesting that each harmonic map constructed in Proposition 1.1 has constant energy density everywhere, the proof of which depends on the characterization of tangent map of the focal map (c.f. pp.245 in [CR85]).

Example 4.1. As we mentioned in the introduction, Cartan classified all isoparametric hypersurfaces in unit spheres with three distinct principal curvatures (see, for example, pp.296297 in [CR85]). More precisely, such an isoparametric hypersurface must be a tube of constant radius over a standard Veronese embedding of a projective plane $\mathbb{F} P^{2}$ into $S^{3 m+1}(1)$, where $\mathbb{F}=\mathbb{R}, \mathbb{C}, \mathbb{H}$ (quaternions), $\mathbb{O}$ (Cayley numbers) for $m=1,2,4,8$, respectively. Let $f: S^{3 m+1}(1) \rightarrow \mathbb{R}$ be the restriction to $S^{3 m+1}(1)$ of the corresponding Cartan-Münzner polynomial. Then $M^{3 m}=f^{-1}(0)$ is the minimal isoparametric hypersurface with three distinct constant principal curvatures $\cot \frac{\pi}{6}, \cot \frac{\pi}{2}, \cot \frac{5 \pi}{6}$ of the same multiplicity $m$ with respect to $\xi=\nabla f /|\nabla f|$, where $\nabla f$ is the gradient of $f$ on $S^{3 m+1}(1)$, and $M_{ \pm}=f^{-1}( \pm 1)$ is isometric to $\mathbb{F} P^{2}$. Define a focal map $\varphi_{\pi / 2}: M \rightarrow M_{-}$by

$$
\varphi_{\pi / 2}(x)=(\cos \pi / 2) x+(\sin \pi / 2) \xi=\xi \quad \text { for } \quad x \in M \subset S^{3 m+1}(1) .
$$

It follows from a direct calculation that the focal map $\varphi_{\pi / 2}: M \rightarrow M_{-}$is a horizontally conformal submersion. In fact(c.f.[GT13]),

$$
\left|\left(\varphi_{\pi / 2}\right)_{*}(X)\right|=\sqrt{3}|X|, \forall X \in\left(\operatorname{Ker}\left(\varphi_{\pi / 2}\right)_{*}\right)^{\perp} \subset T M .
$$


Moreover, the fibers of $\varphi_{\pi / 2}$ are all totally geodesic in $M$. In one word, these facts show that the focal map $\varphi_{\pi / 2}: M \rightarrow M_{-}$is a harmonic morphism by Proposition 1.1 and Remark 4.1

For isoparametric hypersurfaces in unit spheres of OT-FKM type, more harmonic maps are constructed as follows. Let $\left\{P_{0}, \cdots, P_{m}\right\}$ be a symmetric Clifford system on $R^{2 l}$ as before. Then it defines a family of isoparametric hypersurfaces of OT-FKM type and two focal submanifolds $M_{+}, M_{-}$in $S^{2 l-1}$ of codimension $m+1, l-m$, respectively. For any $P \in \Sigma\left(P_{0}, \ldots, P_{m}\right)$, set $\xi=P x$ for $x \in M_{+}$, which is a global unit normal vector field on $M_{+}$in $S^{2 l-1}(1)$. Associated with $\xi$, we define two maps

$$
\phi: M_{+} \rightarrow M_{-}, \psi: M_{+} \rightarrow M
$$

by $\phi(x)=\frac{1}{\sqrt{2}}(x+\xi)$, and $\psi(x)=\cos t x+\sin t \xi$, where $M$ is any isoparametric hypersurface in the family and $t=\operatorname{dist}\left(M_{+}, M\right)$, the spherical distance between $M_{+}$and $M$. Clearly the maps are well defined.

Proposition 4.1. Both of the maps $\phi: M_{+} \rightarrow M_{-}$and $\psi: M_{+} \rightarrow M$ are harmonic maps.

Proof. The proof is similar to that of Theorem 1.4 By Takahashi Theorem and Lemma4.1, it follows that $\phi$ and $\psi$ are harmonic.

Remark 4.4. It is not difficult to prove that $\phi$ and $\psi$ are not harmonic morphisms. Moreover, $\psi$ is a section of the focal map (fibration) from $M$ to $M_{+}$and the map $\psi: M_{+} \rightarrow M$ is a smooth embedding.

Whenever the Clifford system $P_{0}, \cdots, P_{m}$ can be extended to a Clifford system $P_{0}, \cdots, P_{m}$, $P_{m+1}$ on $\mathbb{R}^{2 l}$, one can define $\eta=P_{m+1} x$ for $x \in M_{-}$in $S^{2 l-1}(1)$. In fact, $\eta$ is a global unit normal vector field on $M_{-}$. Associated with $\eta$, we can also define two maps

$$
\tilde{\phi}: M_{-} \rightarrow M_{+}, \tilde{\psi}: M_{-} \rightarrow M
$$

by $\tilde{\phi}(x)=\frac{1}{\sqrt{2}}(x+\eta)$, and $\tilde{\psi}(x)=\cos s x+\sin s \eta$, where $s=\operatorname{dist}\left(M_{-}, M\right)$, the spherical distance between $M_{-}$and $M$.

Proposition 4.2. Both of the maps $\tilde{\phi}: M_{-} \rightarrow M_{+}$and $\tilde{\psi}: M_{-} \rightarrow M$ are harmonic maps.

Proof. The proof is analogous to that of Proposition 4.1 and is omitted.

Remark 4.5. It is not difficult to prove that $\tilde{\phi}$ and $\tilde{\psi}$ are not harmonic morphisms. Moreover, the map $\tilde{\psi}: M_{-} \rightarrow M$ is a smooth embedding. In fact, $\tilde{\psi}$ is a section of the focal map (fibration) from $M$ to $M_{-}$.

We now study the stability of harmonic maps. Recall that a compact Riemannian manifold $M$ is called harmonically unstable, if there exists neither nonconstant stable harmonic map from $M$ to any Riemannian manifold nor from any compact Riemannian manifold to $M$ (c.f. [Oh86]). A significant result states that if $M$ is harmonically unstable then $\pi_{1}(M)=0$ and $\pi_{2}(M)=0$. For minimal submanifolds in unit spheres, Ohnita obtained the following elegant result. 
Theorem 4.1. [Oh86] Let $M$ be an m-dimensional closed minimal submanifold in a unit sphere $S^{n}(1)$. If the Ricci curvature $\rho$ of $M$ satisfies $\rho>m / 2$, then $M$ is harmonically unstable.

Using this theorem, Ohnita also investigated the harmonically unstability of minimal isoparametric hypersurfaces in unit spheres. As mentioned before, the focal submanifolds of isoparametric hypersurfaces are minimal in the unit sphere. Hence we get the following proposition by applying Ohnita's theorem.

Proposition 4.3. Given a symmetric Clifford system $\left\{P_{0}, \ldots, P_{m}\right\}$ on $\mathbb{R}^{2 l}$ and consider the corresponding isoparametric hypersurface of OT-FKM type in $S^{2 l-1}(1)$ with $g=4$ and multiplicities $\left(m_{1}, m_{2}\right)=(m, l-m-1)$. For the focal submanifold $M_{+}$of codimension $m+1$ in $S^{2 l-1}(1)$, if $\left(m_{1}, m_{2}\right) \neq(1,1),(1,2),(2,1),(2,3),(4,3),(5,2),(6,1)$ and $(9,6)$, then $M_{+}$is harmonically unstable.

Proof. According to [TY12], for each point $x \in M_{+}$and any unit tangent vector $X \in T_{x} M_{+}$, the Ricci curvature of $M_{+}$is given by

$$
\rho(X, X)=2(l-m-2)+2 \sum_{0 \leq \alpha<\beta \leq m}\left\langle X, P_{\alpha} P_{\beta} x\right\rangle^{2} .
$$

Then the proposition follows from the formula above and Ohnita's theorem.

Remark 4.6. For the exceptional cases in the proposition above, we have

1). $\left(m_{1}, m_{2}\right)=(1,1)$ : Since $M_{+}^{3}$ is diffeomorphic to $\mathrm{SO}(3)$ (see pp. 301 of [CR85]), $\pi_{1}\left(M_{+}^{3}\right)=\mathbb{Z}_{2}$. Hence $M_{+}^{3}$ is not harmonically unstable.

2). $\left(m_{1}, m_{2}\right)=(1,2)$ : Since $M_{+}^{5}$ is diffeomorphic to the unit tangent bundle of $S^{3}$ (see pp. 301 of [CR85]), which is diffeomorphic to $S^{2} \times S^{3}, \pi_{2}\left(M_{+}^{5}\right)=\pi_{2}\left(S^{2} \times S^{3}\right)=\mathbb{Z}$. Hence $M_{+}^{5}$ is not harmonically unstable.

3). $\left(m_{1}, m_{2}\right)=(2,1)$ : Since $M_{+}^{4}$ is diffeomorphic to $\left(S^{1} \times S^{3}\right) / \mathbb{Z}_{2}$ (see pp. 303 of [CR85]), which is in turn diffeomorphic to $S^{1} \times S^{3}, \pi_{1}\left(M_{+}^{4}\right)=\pi_{1}\left(S^{1} \times S^{3}\right)=\mathbb{Z}$. Hence $M_{+}^{4}$ is not harmonically unstable.

4). $\left(m_{1}, m_{2}\right)=(5,2)$ : Since $\pi_{1}\left(M_{+}^{9}\right)=0$, by Hurwitz isomorphism, $\pi_{2}\left(M_{+}^{9}\right)=H_{2}\left(M_{+}, \mathbb{Z}\right)=$ $\mathbb{Z}$ (c.f. [Mü80]). Hence $M_{+}^{9}$ is not harmonically unstable.

5). $\left(m_{1}, m_{2}\right)=(6,1)$ : Similar to the case 3$), \pi_{1}\left(M_{+}^{8}\right)=\mathbb{Z}$. Hence $M_{+}^{8}$ is not harmonically unstable.

6). $\left(m_{1}, m_{2}\right)=(4,3)$ and the homogeneous case: For each point $x \in M_{+}^{10}$ and any unit tangent vector $X \in T_{x} M_{+}^{10}$, the Ricci curvature

$$
\rho(X, X)=4+2 \sum_{0 \leq \alpha<\beta \leq 4}\left\langle X, P_{\alpha} P_{\beta} x\right\rangle^{2}=6>10 / 2,
$$

since $\left\{P_{\alpha} P_{\beta} x \mid 0 \leq \alpha<\beta \leq 4\right\}$ is an orthonormal basis of $T_{x} M_{+}^{10}$ (c.f. [QTY13]). Hence $M_{+}^{10}$ is harmonically unstable by Ohnita's Theorem. 
There are still three cases we have not determined, i.e., $\left(m_{1}, m_{2}\right)=(2,3),(4,3)$ and the inhomogeneous case, or $(9,6)$.

Remark 4.7. As a result of Proposition 4.3 and Remark 4.6, there exist unstable harmonic maps among the ones we constructed in Proposition 1.1, 4.1 and 4.2.

\section{Counterexamples to Leung's conjectures}

This section will use the expansion formula of Cartan-Münzner polynomial and the isoparametric triple system to prove Theorem 1.7, providing infinitely many counterexamples to two conjectures of Leung [Le91] on minimal submanifolds in unit spheres.

\section{Proof of Theorem 1.7:}

Proof. Let $M^{n}$ be an isoparametric hypersurface in $S^{n+1}(1)$ with $g=4$ and multiplicities $\left(m_{1}, m_{2}\right)$, and denote by $M_{+}$and $M_{-}$the focal submanifolds of $M^{n}$ in $S^{n+1}$ with dimension $m_{1}+2 m_{2}$ and $2 m_{1}+m_{2}$ respectively. Note $n=2\left(m_{1}+m_{2}\right)$. Assume $F$ is the associated CartanMünzner isoparametric polynomial of degree four so that $M_{+}$is defined by $F^{-1}(1) \cap S^{n+1}(1)$. To complete the proof of this theorem, we only need to consider $M_{+}$, since if $F$ is changed to $-F, M_{+}$is changed to $M_{-}$. Given $x \in M_{+}$, choose an orthonormal basis $\xi_{\alpha}, \alpha=0,1, \ldots, m_{1}$ for the normal space of $M_{+}$in $S^{2 l-1}(1)$ at the point $x$. Let $A_{\alpha}, \alpha=0,1, \ldots, m_{1}$, be the corresponding shape operators. For any vector $X \in T_{x} M_{+}$, one has $|B(X, X)|^{2}=\sum_{\alpha=0}^{m_{1}}\left\langle A_{\alpha} X, X\right\rangle^{2}$, where $B$ is the second fundamental form of $M_{+}$in $S^{2 l-1}(1)$.

For our purpose, we first recall a formulation of the Cartan-Münzner polynomial $F$ in terms of the second fundamental forms of the focal submanifolds, developed by Ozeki and Takeuchi (see pp. 52 of [CCJ07] and also [OT75]). For $x \in M_{+}$, and an orthonormal basis $\left\{\xi_{\alpha} \mid \alpha=0,1, \ldots, m_{1}\right\}$ of the normal space of $M_{+}$in $S^{n+1}(1)$ at $x$, one can introduce the quadratic homogeneous polynomials $p_{\alpha}(y):=\left\langle A_{\alpha} y, y\right\rangle$, for $0 \leq \alpha \leq m_{1}$, where $y$ is tangent to $M_{+}$at $x$. The Cartan-Münzner polynomial $F$ is related to $p_{\alpha}$ as follows,

$$
\begin{aligned}
F(t x+y+w)= & t^{4}+\left(2|y|^{2}-6|w|^{2}\right) t^{2}+8\left(\sum_{\alpha=0}^{m_{1}} p_{\alpha}(y) w_{\alpha}\right) t \\
& +|y|^{4}-2 \sum_{\alpha=0}^{m_{1}}\left(p_{\alpha}(y)\right)^{2}+8 \sum_{\alpha=0}^{m_{1}} q_{\alpha}(y) w_{\alpha} \\
& +2 \sum_{\alpha, \beta=0}^{m_{1}}\left\langle\nabla p_{\alpha}, \nabla p_{\beta}\right\rangle w_{\alpha} w_{\beta}-6|y|^{2}|w|^{2}+|w|^{4},
\end{aligned}
$$

where the homogeneous polynomial of degree three, $q_{\alpha}(y)$, are the components of the third fundamental form of $M_{+}$, and $w=\sum_{\alpha=0}^{m_{1}} w_{\alpha} \xi_{\alpha}$.

By the expansion formula above, we observe that for any $X \in T_{x} M_{+}$

$$
F(X)=|X|^{4}-2 \sum_{\alpha=0}^{m_{1}}\left(p_{\alpha}(X)\right)^{2}=|X|^{4}-2|B(X, X)|^{2} .
$$


Hence, $|B(X, X)|^{2}=\frac{|X|^{4}-F(X)}{2}$.

Next, we will give an investigation into the possible value of $|B(X, X)|^{2}$, from which Theorem 1.7 follows immediately. To do it, we use the isoparametric triple system introduced by Dorfmeister and Neher, following the way in [Im08]. Let $x^{\prime}$ be a unit vector normal to the tangent space $T_{x} M_{+}$in $T_{x} S^{n+1}$. Then the great circle $S$ through $x$ and $x^{\prime}$ intersects the isoparametric hypersurface and two focal submanifolds orthogonally at each intersection point. The set $S \cap M_{+}$consists of the four points $\pm x$ and $\pm x^{\prime}$, and the set $S \cap M_{-}$consists of the four points $\pm y$ and $\pm y^{\prime}$, where $\sqrt{2} x=y-y^{\prime}$ and $\sqrt{2} x^{\prime}=y+y^{\prime}$. There are orthogonal Peirce decompositions

$$
\mathbb{R}^{2 m_{1}+2 m_{2}+2}=\operatorname{Span}\{x\} \oplus V_{-3}(x) \oplus V_{1}(x)=\operatorname{Span}\{y\} \oplus V_{3}(y) \oplus V_{-1}(y),
$$

where $V_{-3}(x)=T_{x}^{\perp} M_{+}$, the normal space of $M_{+}$in $S^{n+1}(1)$ at $x, V_{1}(x)=T_{x} M_{+}, V_{3}(y)=T_{y}^{\perp} M_{-}$, the normal space of $M_{-}$in $S^{n+1}(1)$ at $y$, and $V_{-1}(y)=T_{y} M_{-}$, the so-called Peirce spaces. Furthermore, as one of the main results in [Im08], Immervoll gave a more subtle orthogonal decomposition as

$$
\mathbb{R}^{2 m_{1}+2 m_{2}+2}=\operatorname{Span}(S) \oplus V_{-3}^{\prime}(x) \oplus V_{-3}^{\prime}\left(x^{\prime}\right) \oplus V_{3}^{\prime}(y) \oplus V_{3}^{\prime}\left(y^{\prime}\right)
$$

where the subspaces $V_{-3}^{\prime}(x), V_{-3}^{\prime}\left(x^{\prime}\right), V_{3}^{\prime}(y)$ and $V_{3}^{\prime}\left(y^{\prime}\right)$ are defined by $V_{-3}(x)=\operatorname{Span}\left\{x^{\prime}\right\} \oplus$ $V_{-3}^{\prime}(x), V_{-3}\left(x^{\prime}\right)=\operatorname{Span}\{x\} \oplus V_{-3}^{\prime}\left(x^{\prime}\right), V_{3}(y)=\operatorname{Span}\left\{y^{\prime}\right\} \oplus V_{3}^{\prime}(y)$ and $V_{3}\left(y^{\prime}\right)=\operatorname{Span}\{y\} \oplus V_{3}^{\prime}\left(y^{\prime}\right)$. It follows from the two decompositions above that

$$
T_{x} M_{+}=V_{-3}^{\prime}\left(x^{\prime}\right) \oplus V_{3}^{\prime}(y) \oplus V_{3}^{\prime}\left(y^{\prime}\right) .
$$

Now taking a unit vector $X_{1}$ in $V_{3}^{\prime}(y)$, we see that $X_{1} \in M_{-}$. Actually, $S^{2 l-1}(1) \cap V_{3}(y) \subset$ $M_{-}$. Similarly, any unit vector $X_{1}$ in $V_{3}^{\prime}\left(y^{\prime}\right)$ also belongs to $M_{-}$. Hence, for any unit vectors $X_{1} \in V_{3}^{\prime}(y) \oplus V_{3}^{\prime}\left(y^{\prime}\right)$, we have $F\left(X_{1}\right)=-1$, and thus $\left|B\left(X_{1}, X_{1}\right)\right|^{2}=\frac{1-F\left(X_{1}\right)}{2}=1$. On the other hand, for any unit vector $X_{0} \in V_{-3}^{\prime}\left(x^{\prime}\right)$, we can see that $X_{0} \in M_{+}$and $F\left(X_{0}\right)=1$. Therefore, $\left|B\left(X_{0}, X_{0}\right)\right|^{2}=\frac{1-F\left(X_{0}\right)}{2}=0$. We have proved that $M_{+}^{m_{1}+2 m_{2}}$ is a minimal submanifold in $S^{n+1}(1)$ with $\sigma\left(M_{+}\right)=1$.

Lastly, according to [Mü80], the cohomology ring of $M_{+}$is different from that of $S^{m_{1}+2 m_{2}}$, and thus $M_{+}$is not homeomorphic to $S^{m_{1}+2 m_{2}}$.

Now, the proof is complete.

Acknowledgements . The authors would like to thank Professors Y. Ohnita and E. Loubeau for useful comments on the stability of harmonic maps, and thank Prof. Jiagui Peng for helpful advices during the preparation of the paper. Thanks are also due to Prof. Weiping Zhang for presenting the second author with the book [ER93] from Paris. Finally, the authors are very grateful to the referees for useful comments and suggestions.

\section{REFERENCES}

[BM77] B. L. Beers and R. S. Millman, The spectra of the Laplace-Beltrami operator on compact, semisimple Lie groups, Amer. J. Math., 99(1977), 801-807. 
[CCJ07] T. E. Cecil, Q. S. Chi and G. R. Jensen, Isoparametric hypersurfaces with four principal curvatures, Ann. Math., 166(2007), 1-76.

[CR85] T. E. Cecil and P. T. Ryan, Tight and taut immersions of manifolds, Research Notes in Math. 107, Pitman, London, 1985.

[CF78] I. Chavel and E. A. Feldman, Spectra of domains in compact manifolds, J. Funct. Anal., 30(1978), 198-222.

[Ch96] J. Y. Chen, Stable harmonic maps into the complex projective spaces, J. Diff. Geom., 43(1996), 42-65.

[Ch11] Q. S. Chi, Isoparametric hypersurfaces with four principal curvatures, II, Nagoya Math. J.,204(2011), $1-18$.

[Ch13] Q. S. Chi, Isoparametric hypersurfaces with four principal curvatures, III, J. Diff. Geom., 94(2013), 487504.

[EL78] J. Eells and L. Lemaire, A report on harmonic maps, Bull. London Math. Soc., 10(1978), 1-68.

[EL83] J. Eells and L. Lemaire, Selected topics in harmonic maps, C.B.M.S. Regional Conf. Series in Math., Vol. 50, Amer. Math. Soc., Providence, R.I., 1983.

[EL88] J. Eells and L. Lemaire, Another report on harmonic maps, Bull. London Math. Soc., 20(1988), 385-524.

[ER93] J. Eells and A. Ratto, Harmonic maps and minimal immersions with symmetries. Methods of ordinary differential equations applied to elliptic variantional problems, Ann. Math. Studies, vol. 130, Princeton University Press, Princeton, NJ., 1993.

[Fe80] H. D. Fegan, The spectrum of the Laplacian on forms over a Lie group, Pacific J. Math., 90(1980), 373-387.

[FKM81] D. Ferus, H. Karcher, and H. F. Münzner, Cliffordalgebren und neue isoparametrische Hyperflächen, Math. Z. 177 (1981), 479-502.

[Ga86] H. Gauchman, Minimal submanifolds of a sphere with bounded second fundamental form, Trans. A.M.S., 298(1986), 779-791.

[GT13] J. Q. Ge and Z. Z. Tang, Isoparametric functions and exotic spheres, J. für die reine und angew. Math., 683(2013), 163-180.

[HV01] T. Hasanis and T. Vlachos, Ricci curvatures and minimal submanifolds, Pacific J. Math., 197(2001), 1324.

[Im08] S. Immervoll, On the clssification of isoparametric hypersurfaces with four ditinct principal curvatures in spheres, Ann. Math., 168(2008), 1011-1024.

[Le82] P. F. Leung, On stability of harmonic maps, Lecture Notes in Math., Vol. 949, Springer, Berlin, 1982, 122-129.

[Le91] P. F. Leung, Minimal submanifolds in a sphere II, Bull. L.M.S., 23(1991), 387-390.

[Mi13] R. Miyaoka, Isoparametric hypersurfaces with $(g, m)=(6,2)$, Ann. Math., 177(2013), 53-110.

[Mi16] R. Miyaoka, Errata of "Isoparametric hypersurfaces with $(g, m)=(6,2)$ ", to appear in Ann. Math.

[Mü80] H. F. Münzner, Isoparametric hyperflächen in sphären, I and II, Math. Ann., 251(1980), 57-71 and 256(1981), 215-232.

[Mu88] H. Muto, The first eigenvalue of the Laplacian of an isoparametric minimal hypersurface in a unit sphere, Math. Z., 177 (1981), 479-502.

[Oh86] Y. Ohnita, Stability of harmonic maps and standard minimal immersions, Tôhoku Math. J., 38(1986), 259-267.

[Oz81] S. Ozawa, Singular variation of domains and eigenvalues of the Laplacian, Duke Math. J., 48(1981), 767778.

[OT75] H. Ozeki and M. Takeuchi, On some types of isoparametric hypersurfaces in spheres I and II, Tôhoku Math. J., 27(1975), 515-559 and 28(1976), 7-55.

[Pe84] C. K. Peng, Some relations between minimal submanifolds and harmonic maps, Chinese Ann. Math.(Chinese version), 5A(1984), 85-90.

[QT15] C. Qian and Z. Z. Tang, Isoparametric functions on exotic spheres, Adv. Math., 272(2015), 611-629. 
[QTY13] C. Qian, Z. Z. Tang and W. J. Yan, New examples of Willmore submanifolds in the unit sphere via isoparametric functions, II, Ann. Glob. Anal. Geom., 43 (2013), 47-62.

[Ra14] M. Radeschi, Clifford algebras and new singular Riemannian foliations in spheres, Geom. Funct. Anal., 24(2014), 1660-1682.

[So92] B. Solomon, Quartic isoparametric hypersurfaces and quadratic forms, Math. Ann., 293(1992), 387-398.

[TXY14] Z. Z. Tang, Y. Q. Xie and W. J. Yan, Isoparametric foliation and Yau conjecture on the first eigenvalue, II, J. Func. Anal., 266(2014), 6174-6199.

[TY12] Z. Z. Tang and W. J. Yan, New examples of Willmore submanifolds in the unit sphere via isoparametric functions, Ann. Glob. Anal. Geom., 42 (2012), 403-410.

[TY12'] Z. Z. Tang and W. J. Yan, Critical sets of eigenfunctions and Yau conjecture, arXiv:1203.2089, 2012.

[TY13] Z. Z. Tang and W. J. Yan, Isoparametric foliation and Yau conjecture on the first eigenvalue, J. Diff. Geom., 94(2013), 521-540.

[Wa87] Q. M. Wang, Isoparametric functions on Riemannian manifolds. I, Math. Ann., 277(1987), 639-646.

[Wa88] Q. M. Wang, On the topology of Clifford isoparametric hypersurfaces, J. Diff. Geom., 27(1988), 55-66.

[XFX06] H.-W. Xu, W. Fang and F. Xiang, A generalization of Gauchman's rigidity theorem, Pacific J. Math., 228(2006), 185-199.

School of Mathematics and Statistics, Beijing Institute of Technology, Beijing 100081, P.R. China

E-mail address: 6120150035@bit.edu.cn

School of Mathematical Sciences, Laboratory of Mathematics and Complex Systems, Beijing Normal UniVERSITY, BEIJING 100875, P.R.CHINA

E-mail address: zztang@bnu.edu.cn 